\title{
Molecular globules in the Veil bubble of Orion
} IRAM $30 \mathrm{~m}{ }^{12} \mathrm{CO},{ }^{13} \mathrm{CO}$, and $\mathrm{C}^{18} \mathrm{O}(2-1)$ expanded maps of Orion $\mathrm{A}^{\star}$

\author{
J. R. Goicoechea ${ }^{1}$, C. H. M. Pabst ${ }^{2}$, S. Kabanovic ${ }^{3}$, M. G. Santa-Maria ${ }^{1}$, N. Marcelino ${ }^{1}$, A. G. G. M. Tielens ${ }^{2}$,

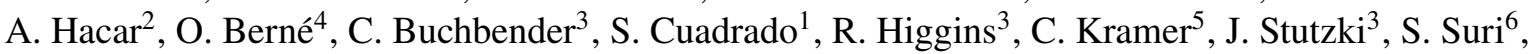 \\ D. Teyssier ${ }^{7}$, and M. Wolfire ${ }^{8}$
}

\footnotetext{
${ }^{1}$ Instituto de Física Fundamental (CSIC), Calle Serrano 121-123, 28006 Madrid, Spain e-mail: javier.r.goicoechea@csic.es

${ }^{2}$ Leiden Observatory, Leiden University, Leiden, The Netherlands

${ }^{3}$ I. Physikalisches Institut der Universität zu Köln, Cologne, Germany

${ }^{4}$ IRAP, Université de Toulouse, CNRS, CNES, Université Paul Sabatier, Toulouse, France

${ }^{5}$ Institut de Radioastronomie Millimétrique (IRAM), Grenoble, France

${ }^{6}$ Max Planck Institute for Astronomy, Heidelberg, Germany

7 Telespazio Vega UK Ltd. for ESA/ESAC, Madrid, Spain

${ }^{8}$ University of Maryland, Astronomy Department, College Park, MD, USA
}

Received 7 January 2020 / Accepted 27 April 2020

\begin{abstract}
Strong winds and ultraviolet (UV) radiation from O-type stars disrupt and ionize their molecular core birthplaces, sweeping up material into parsec-size shells. Owing to dissociation by starlight, the thinnest shells are expected to host low molecular abundances and therefore little star formation. Here, we expand previous maps made with observations using the IRAM $30 \mathrm{~m}$ telescope (at $11^{\prime \prime} \simeq 4500 \mathrm{AU}$ resolution) and present square-degree ${ }^{12} \mathrm{CO}$ and ${ }^{13} \mathrm{CO}(J=2-1)$ maps of the wind-driven "Veil bubble" that surrounds the Trapezium cluster and its natal Orion molecular core (OMC). Although widespread and extended CO emission is largely absent from the Veil, we show that several CO "globules" exist that are blueshifted in velocity with respect to OMC and are embedded in the [C II] $158 \mu \mathrm{m}-$ bright shell that confines the bubble. This includes the first detection of quiescent $\mathrm{CO}$ at negative local standard of rest velocities in Orion. Given the harsh UV irradiation conditions in this translucent material, the detection of CO globules is surprising. These globules are small $\left(R_{\mathrm{g}}=7100 \mathrm{AU}\right)$, not massive $\left(M_{\mathrm{g}}=0.3 M_{\odot}\right)$, and are moderately dense: $n_{\mathrm{H}}=4 \times 10^{4} \mathrm{~cm}^{-3}$ (median values). They are confined by the external pressure of the shell, $P_{\text {ext }} / k \gtrsim 10^{7} \mathrm{~cm}^{-3} \mathrm{~K}$, and are likely magnetically supported. They are either transient objects formed by instabilities or have detached from pre-existing molecular structures, sculpted by the passing shock associated with the expanding shell and by UV radiation from the Trapezium. Some represent the first stages in the formation of small pillars, others of isolated small globules. Although their masses $\left(M_{\mathrm{g}}<M_{\text {Jeans }}\right)$ do not suggest they will form stars, one globule matches the position of a known young stellar object. The lack of extended CO in the "Veil shell" demonstrates that feedback from massive stars expels, agitates, and reprocesses most of the disrupted molecular cloud gas, thereby limiting the star-formation rate in the region. The presence of molecular globules is a result of this feedback.
\end{abstract}

Key words. galaxies: ISM - HII regions - ISM: bubbles - ISM: clouds - ISM: individual objects: Orion

\section{Introduction}

Massive stars dominate the injection of UV radiation into the interstellar medium (ISM) and of mechanical energy through stellar winds and supernova explosions. The energy and momentum injected by photoionization, radiation pressure, and stellar winds from young O-type stars ionize and disrupt their natal molecular cloud cores, creating $\mathrm{H}$ II regions and blowing parsecsize bubbles enclosed by shells of denser swept-up material (e.g., Weaver et al. 1977; Churchwell et al. 2006; Deharveng et al. 2010). These feedback processes may locally regulate the formation of new stars, and globally drive the evolution of the ISM in galaxies as a whole (e.g., Krumholz et al. 2014; Rahner et al. 2017; Haid et al. 2018).

The iconic Extended Orion Nebula (M42) is photoionized by UV photons emitted mainly from the most massive

\footnotetext{
* Based on IRAM $30 \mathrm{~m}$ telescope observations. IRAM is supported by INSU/CNRS (France), MPG (Germany), and IGN (Spain).
}

star in the Trapezium cluster, $\theta^{1}$ Ori $\mathrm{C}$ (type $\mathrm{O} 7 \mathrm{~V}$ and $Q_{\mathrm{Ly}} \simeq$ $6 \times 10^{48}$ photons s $^{-1}$; e.g., O’Dell 2001; Simón-Díaz et al. 2006; Gravity Collaboration 2018). In addition, the characteristic bubble-shape ( $\sim \mathrm{pc}$ in diameter) and overall dynamics of M 42 seem ultimately driven by the strong wind emanating from $\theta^{1}$ Ori C (Güdel et al. 2008; Pabst et al. 2019). The foreground material that surrounds the Trapezium and its natal molecular core-1 (OMC-1, located behind the cluster, e.g., Genzel \& Stutzki 1989; Bally 2008) is generically known as the "Veil" (O'Dell 2001; van der Werf et al. 2013; Troland et al. 2016; Abel et al. 2019). The "Veil bubble" is filled with an X-rayemitting (wind-shocked) million-degree plasma (Güdel et al. 2008). As delineated by the $\mathrm{H} \alpha$ emission, the inside of this bubble is also an $\mathrm{HII}$ region photo-ionized by UV radiation from $\theta^{1}$ Ori C. In the far side, the bubble is confined by dense molecular gas at the surface of the OMC (Rodríguez-Franco et al. 1998; Goicoechea et al. 2019) and, in the near side, by an expanding half-shell of warm gas $\left(T_{\mathrm{k}} \simeq 100 \mathrm{~K}\right)$ and dust (see sketch in 

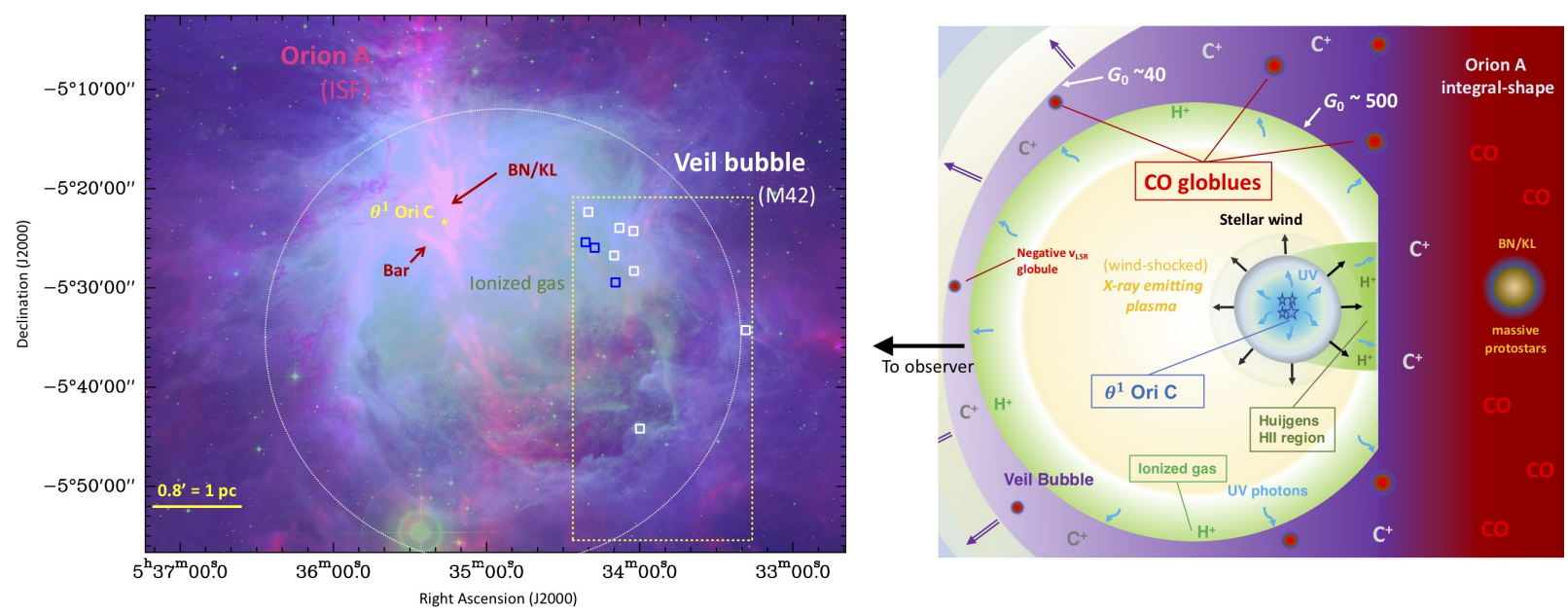

Fig. 1. Left: extended Orion Nebula (M 42), part of the integral-shape filament in Orion A (reddish colors), and the Veil bubble (delineated by a dotted circle) filled with ionized gas (greenish). Red: SPIRE $500 \mu \mathrm{m}$ image (cold dust from the background molecular cloud). Blue: PACS $70 \mu \mathrm{m}$ image (warm dust). Green: H $\alpha$ image adapted from ESO's Second Digitized Sky Survey (DSS2, see Pabst et al. 2020). The yellow star corresponds to the position of $\theta^{1}$ Ori $\mathrm{C}$ in the Trapezium cluster. Here we focus on the $17.5^{\prime} \times 34.5^{\prime}$ area enclosed by the dotted box. The blue and white squares mark the position of $\mathrm{CO}$ globules detected in the expanding shell that confines the bubble (blue squares for the negative- $v_{\mathrm{LSR}}$ globules). Right: sketch of the region, not at scale, adapted from Pabst et al. (2019).

Fig. 1). The swept-up material in the shell is very bright in the ${ }^{2} P_{3 / 2}-{ }^{2} P_{1 / 2}$ fine-structure emission of $\mathrm{C}^{+}$(the well-known [C II] $158 \mu \mathrm{m}$ line). In Pabst et al. (2019) we obtained squaredegree velocity-resolved images of the [C II] $158 \mu \mathrm{m}$ emission with SOFIA, and showed that the mechanical energy from the stellar wind from $\theta^{1}$ Ori C (terminal velocity of $\sim 2500 \mathrm{~km} \mathrm{~s}^{-1}$; Stahl et al. 1996) is effectively converted into kinetic energy of the shell. This stellar wind causes more disruption of OMC-1 (well before any supernova explosion) than photo-ionization or photo-evaporation (Pabst et al. 2019).

Although not the most numerous or massive star cluster in the Milky Way, the proximity of the Orion nebula, the Trapezium stars, and the molecular core OMC-1 (the closest core that hosts ongoing massive-star formation) enables us to study star formation and stellar feedback in great spatial detail (like in our previous works, here we adopt $\sim 414$ pc; e.g., Menten et al. 2007).

As in other thin shells around high-mass stars, the intense UV radiation in M 42 suggests very low molecular abundances in the "Veil shell". Indeed, previous observations of the line of sight toward the Trapezium stars imply small columns of material in the Veil (O'Dell 2001), 1-2 mag of visual extinction $\left(A_{\mathrm{V}}\right)$, and also low molecular gas fractions $\left(x\left(\mathrm{H}_{2}\right) / x(\mathrm{H})<10^{-4}\right.$; where $x$ is the abundance with respect to $\mathrm{H}$ nuclei, Abel et al. 2006).

$\mathrm{CO}$, the second most abundant molecule in the ISM, had not been detected toward the Veil before. As the emission from cold $\mathrm{H}_{2}$ is not directly observable either (e.g., Bolatto et al. 2013), the lack of detectable $\mathrm{CO}$ emission poses uncertain constraints on the measurable mass of molecular material that escapes detection in wide-field CO radio surveys (Grenier et al. 2005; Planck Collaboration XIX 2011). This extended "CO-dark" molecular gas (when the CO column density, $N(\mathrm{CO})$, is too low to be detected) may represent $30 \%$ of the molecular gas mass in the Milky Way (Grenier et al. 2005; Wolfire et al. 2010). This fraction can be much higher in the ISM of low-metallicity galaxies (Madden et al. 1997) characterized by a higher penetration of stellar UV radiation. In this context, the Orion's Veil is an interesting nearby template to study the origin and properties of the vast neutral halos that surround many star-forming regions.

This paper is organized as follows. In Sect. 2 we describe the new CO $(J=2-1)$ mapping observations. In Sect. 3 we present the main observational result of this work, namely the detection of globules embedded in the shell that confines the Veil bubble. In Sect. 4 we analyze the environment and the properties of these globules, and in Sect. 5 we discuss their origin and evolution.

\section{Observations and data reduction}

We obtained new ${ }^{12} \mathrm{CO},{ }^{13} \mathrm{CO}$, and $\mathrm{C}^{18} \mathrm{O}(J=2-1)$ fully sampled maps of Orion A using the IRAM $30 \mathrm{~m}$ telescope (Pico Veleta, Spain). The bright central region $\left(1^{\circ} \times 0.8^{\circ}\right)$ around OMC-1 was originally mapped in 2008 (Berné et al. 2014) with the multi-beam receiver HERA at $0.4 \mathrm{~km} \mathrm{~s}^{-1}$ resolution (Schuster et al. 2004). In order to cover the larger area (1.2 square-degree) mapped by us in the [C II] $158 \mu \mathrm{m}$ line with SOFIA/GREAT at comparable angular resolution (Legacy Program led by A. G. G. M. Tielens) we started to expand the CO maps using EMIR (Carter et al. 2012) and FFTS backends at the $30 \mathrm{~m}$ telescope. These new observations of fainter regions in Orion A were carried out in October 2018, March 2019, November 2019, and February 2020, so far employing 100 h of telescope time, and are part of the Large Program "Dynamic and Radiative Feedback of Massive Stars" (PI: J. R. Goicoechea).

The ${ }^{12} \mathrm{CO} J=2-1(230.5 \mathrm{GHz}),{ }^{13} \mathrm{CO} J=2-1(220.4 \mathrm{GHz})$, and $\mathrm{C}^{18} \mathrm{O} J=2-1(219.5 \mathrm{GHz})$ lines were simultaneously mapped with EMIR, providing an instantaneous bandwidth of $16 \mathrm{GHz}$ per polarization, in combination with FFTS backends at $200 \mathrm{kHz}$ resolution $\left(\sim 0.25 \mathrm{~km} \mathrm{~s}^{-1}\right)$. The half power beam width (HPBW) at $230.5 \mathrm{GHz}$ is $10.7^{\prime \prime}$. The observing strategy consisted in mapping boxes of $\sim 534^{\prime \prime} \times 534^{\prime \prime}$ size using the on-the-fly (OTF) technique. Each box was mapped through 16 rectangular tiles of 8 OTF scans each, followed by a calibration measurement. In total, we employed about $12 \mathrm{~min}$ per tile. The scanning velocity was $\sim 9^{\prime \prime} \mathrm{s}^{-1}$ and the dump time was $\sim 0.4 \mathrm{~s}$. This combination provided three dumps per beam along the scanning direction. The separation between two successive raster lines was 4.2" (roughly beam/2.5) with the scanning direction reversed after each raster line (i.e., zigzag scanning mode). To avoid edgeeffects between consecutive boxes, we overlapped the observation of each box edge by slightly more than one beam. In order to decrease stripping, each box was mapped twice, scanning in 

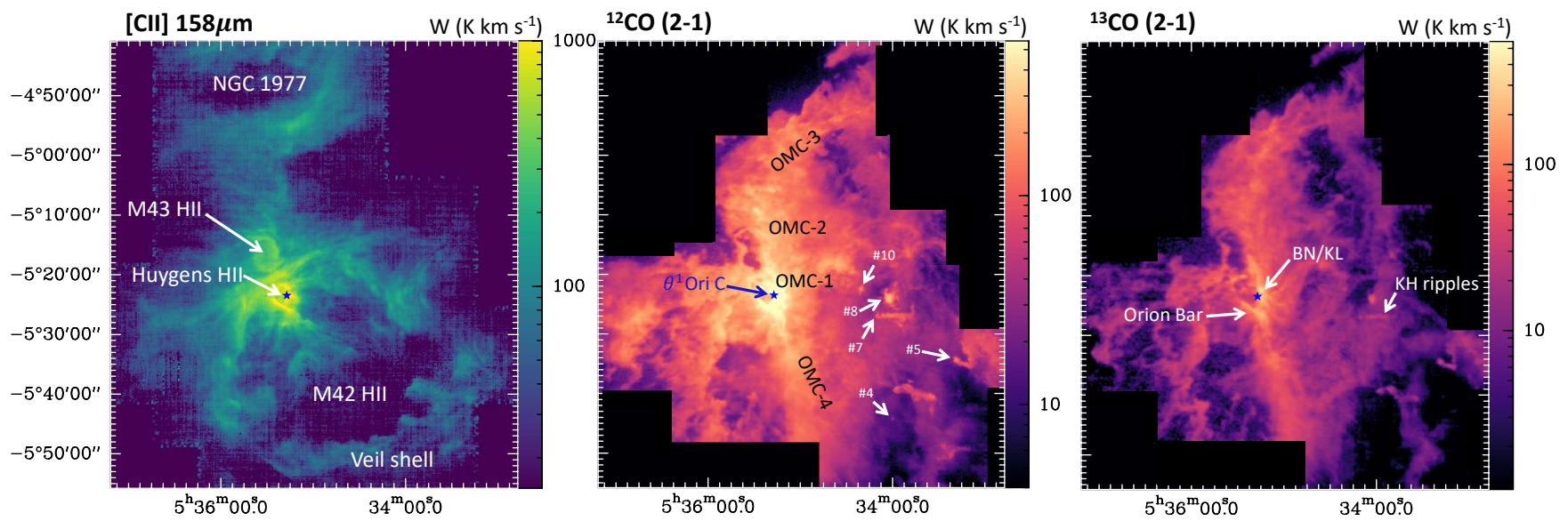

Fig. 2. [C II] $158 \mu \mathrm{m},{ }^{12} \mathrm{CO}(2-1)$, and ${ }^{13} \mathrm{CO}(2-1)$ integrated line intensity maps of the central square-degree region of Orion A. Some of the main structures and components discussed in this work are labelled. The [C II] $158 \mu \mathrm{m}$ map was observed by SOFIA/UPGREAT at an agular resolution of $16^{\prime \prime}$ (Pabst et al. 2019). The ${ }^{12} \mathrm{CO}$ and ${ }^{13} \mathrm{CO}$ maps, observed with the IRAM $30 \mathrm{~m}$ telescope, have a resolution of $11^{\prime \prime}$.

two orthogonal directions. We used a common reference position that we call REF, at an offset $\left(-60^{\prime},-30^{\prime}\right)$ from the map center. We obtained deep spectra of the REF position using the frequency switching mode. These spectra do not show $\mathrm{CO}$ emission at the rms level of the map. The REF position was observed for $10 \mathrm{~s}$ after each raster line (taking $\sim 60 \mathrm{~s}$ ) following the pattern REF-OTF-OTF-REF. Pointing was checked every $2 \mathrm{~h}$ and focus every $4 \mathrm{~h}$ or after sunset. We started every day with a pointed observation of the Orion Bar to cross-check the intensity of the CO lines. We estimate an absolute intensity error of about $15 \%$.

The new data were first calibrated in the $T_{\mathrm{A}}^{*}$ scale, and were then corrected for atmospheric absorption and spillover losses using the chopper-wheel method (Penzias \& Burrus 1973). Most of these observations were made under very good winter conditions (less than $2 \mathrm{~mm}$ of atmospheric precipitable water vapor). The receiver and system temperatures were typically $\sim 100 \mathrm{~K}$ and $\sim 200-300 \mathrm{~K}$, respectively. For emission sources of brightness temperature $T_{\mathrm{b}}(v)$ at a given velocity channel, the main-beam temperature is the most appropriate intensity scale (i.e., $T_{\mathrm{mb}}(v) \simeq$ $T_{\mathrm{b}}(v)$ ) when the emission source fills the main beam of the telescope. Assuming a disk-like emission source of uniform $T_{\mathrm{b}}$ and angular size varying from $\sim 25^{\prime \prime}$ to $90^{\prime \prime}$, the ratio $T_{\mathrm{mb}} / T_{\mathrm{b}}$ at $230 \mathrm{GHz}$ goes from 1.0 to 1.2 at the IRAM $30 \mathrm{~m}$ telescope (whereas $T_{\mathrm{A}}^{*} / T_{\mathrm{b}}$ goes from 0.4 to 0.5 ; see Online material in Teyssier et al. 2002). Molecular clouds have complicated emission structures, spatially and in velocity, meaning that the $T_{\mathrm{mb}}$ scale is widely used as a good compromise when the emission sources are smaller than the very wide antenna error beams (for the IRAM $30 \mathrm{~m}$ telescope, see Greve et al. 1998). Here we converted the intensity scale from $T_{\mathrm{A}}^{*}$ to $T_{\mathrm{mb}}\left(=T_{\mathrm{A}}^{*} \cdot F_{\mathrm{eff}} / B_{\mathrm{eff}}\right)$ using the main-beam efficiency $B_{\text {eff }}$ and forward efficiency $F_{\text {eff }}$ appropriate for each frequency $\left(B_{\text {eff }}=0.59\right.$ and $F_{\text {eff }}=0.92$ at $230 \mathrm{GHz})$.

Data reduction was carried out with the GILDAS software $^{1}$. A polynomic baseline of order 1 or 2 was subtracted avoiding velocities with molecular emission. Finally, the spectra were gridded into a data cube through a convolution with a Gaussian kernel of approximately one-third the telescope HPBW. The typical $(1 \sigma)$ rms noise level achieved in the map is $0.25 \mathrm{~K}$ per $0.25 \mathrm{~km} \mathrm{~s}^{-1}$ velocity channel. This is typically a factor of more than three deeper than the rms of the large ${ }^{12} \mathrm{CO}(1-0)$ map obtained by merging CARMA interferometric

1 http://www.iram.fr/IRAMFR/GILDAS and NRO $45 \mathrm{~m}$ telescope observations at $10^{\prime \prime} \times 8^{\prime \prime}$ angular resolution (Kong et al. 2018). Although these latter authors detect the stronger positive-velocity globules and other bright structures (their Fig. 8), the sensitivity in our maps allowed us to investigate faint and diffuse $\mathrm{CO}(2-1)$ emission structures and compact globules at local standard of rest (LSR) velocities significantly blueshifted from those of the OMC.

In order to compare with our SOFIA [C II] $158 \mu \mathrm{m}$ map, we merged the older CO HERA observations with the expanded EMIR maps. Thanks to the improved new software, MRTCAL, at the $30 \mathrm{~m}$ telescope with calibration on a finer frequency grid, line calibration has slightly improved since 2017. In addition, telescope efficiencies have slightly changed as well. Hence, we took the new EMIR data as the reference for the CO line intensities. To do that, we re-observed a few common areas and produced scatter plots of the $\mathrm{CO}$ line integrated intensities (HERA vs. EMIR maps). The derived linear slopes deviate by $<15 \%$, and we used this correction factor to scale up the HERA data. Figure 2 shows the (current) extent of the ${ }^{12} \mathrm{CO}$ and ${ }^{13} \mathrm{CO}(2-1)$ merged maps, whereas Fig. 3 zooms into the area of interest for this work.

In order to properly compare the ${ }^{12} \mathrm{CO}(2-1),{ }^{13} \mathrm{CO}(2-1)$, and $[\mathrm{C} \mathrm{II}] 158 \mu \mathrm{m}$ line profiles at the same angular and spectral resolutions, we created cubes convolved, with a Gaussian kernel, to uniform resolutions of $16^{\prime \prime}$ and $0.4 \mathrm{~km} \mathrm{~s}^{-1}$, respectively. The convolved and smoothed $\mathrm{CO}$ maps were used in the line profile analysis (see Sect. 4 and Fig. 5 for the spectra) as well as in the position-velocity diagrams (Figs. 7 and A.2-A.9). The typical rms noise in the smoothed ${ }^{12} \mathrm{CO}(2-1)$ map is $0.16 \mathrm{~K}$ per $0.4 \mathrm{~km} \mathrm{~s}^{-1}$ channel. We used Gaussian fits to extract the line profile parameters of the blueshifted $\mathrm{CO}$ globules: spectral components peaking at LSR velocities lower than those of OMC (i.e., $v_{\mathrm{LSR}}<+(7-10) \mathrm{km} \mathrm{s}^{-1}$; Bally et al. 1987; Berné et al. 2014; Kong et al. 2018). Line fit parameters are tabulated in Tables A.1 and A.2. When appropriate, offsets in arcsec are given with respect to star $\theta^{1}$ Ori C, at $\alpha(2000)=05 \mathrm{~h} 35 \mathrm{~m} 16.46 \mathrm{~s}$ and $\delta(2000)=-05^{\circ} 23^{\prime} 22.8^{\prime \prime}$.

\section{Results}

Figure 2 shows a square-degree area of the Orion A molecular complex. At visible wavelengths, the region is dominated by M42, the extended Orion nebula, ionized by the strong UV 

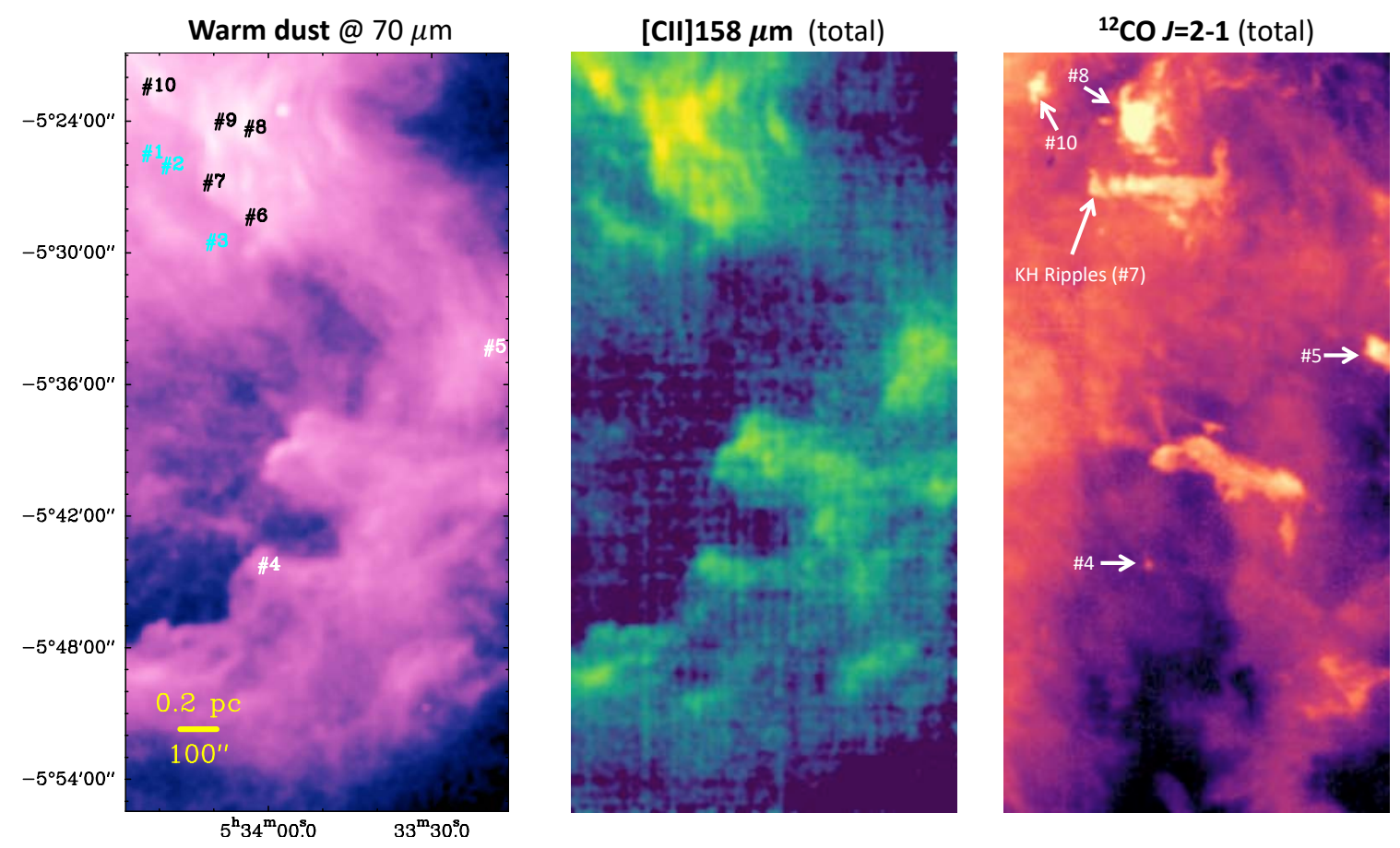

Fig. 3. Zoom on the southwest region of the Veil shell. The first two images show the emission from FUV-heated warm dust and from $\mathrm{C}^{+}$. The rightmost image displays a rather different morphology, mostly dominated by extended CO from the molecular cloud behind the shell. Left panel: PACS $70 \mu \mathrm{m}$ emission at $6^{\prime \prime}$ resolution and positions of the detected CO globules. Middle panel: SOFIA [C II] $158 \mu \mathrm{m}$ intensity map integrated in the $v_{\mathrm{LSR}}=[-7,+20] \mathrm{km} \mathrm{s}^{-1}$ range (Pabst et al. 2019). Right panel: IRAM $30 \mathrm{~m}^{12} \mathrm{CO}(2-1)$ intensity map in the $v_{\mathrm{LSR}}=[-7,+20] \mathrm{km} \mathrm{s} \mathrm{s}^{-1} \mathrm{range}$. We identified blueshifted $\mathrm{CO}$ globules (some of them are labeled) with velocity centroids in the $v_{\mathrm{LSR}}$ range $[-7,+6] \mathrm{km} \mathrm{s}^{-1}$ (see Fig. 4).

radiation field from $\theta^{1}$ Ori $\mathrm{C}$ (e.g., O’Dell 2001). While in this region the [C II] $158 \mu \mathrm{m}$ emission mostly traces UV-illuminated gas around $\mathrm{H}$ II regions, the majority of the ${ }^{12} \mathrm{CO}(2-1)$ and ${ }^{13} \mathrm{CO}(2-1)$ integrated line emission arises from the molecular cloud behind (see sketch Fig. 1). The properties of the main star-forming cores (OMC-1, OMC-2, OMC-3, and OMC-4) have been extensively discussed in previous $\mathrm{CO}$ maps of the region (e.g., Bally et al. 1987; Shimajiri et al. 2011; Buckle et al. 2012; Berné et al. 2014; Kong et al. 2018). Here we focus on $\mathrm{a} \sim 17.5^{\prime} \times 34.5^{\prime}(\sim 2 \mathrm{pc} \times 4 \mathrm{pc})$ region of the bubble (zoomed in Fig. 3) southwest from the Trapezium. The Veil shell is very conspicuous at $70 \mu \mathrm{m}$ (warm grains), in the [C II] $158 \mu \mathrm{m}$ line (Pabst et al. 2019), and in the extended $8 \mu \mathrm{m}$ emission produced by polycyclic aromatic hydrocarbons (PAHs, Fig. 4 left). However, the ${ }^{12} \mathrm{CO}$ integrated line intensity map shows a rather different morphology, which is dominated by emission structures in the background dense molecular cloud. This region of ongoing star formation, part of Orion's integral-shape filament, dominates the $\mathrm{CO}$ emission and peaks at local standard of rest velocities $\left(v_{\mathrm{LSR}}\right)$ around +(7-10) $\mathrm{km} \mathrm{s}^{-1}$ (e.g., Bally et al. 1987; Shimajiri et al. 2011; Buckle et al. 2012; Berné et al. 2014; Kong et al. 2018). At these positive LSR velocities, high-resolution (sub- $\mathrm{km} \mathrm{s}^{-1}$ ) spectra of the [C II] $158 \mu \mathrm{m}$ line display bright emission from the UV-irradiated surface of the dense molecular cloud (Boreiko \& Betz 1996; Ossenkopf et al. 2013; Goicoechea et al. 2015, 2019; Cuadrado et al. 2019).

In addition, the [C II $158 \mu \mathrm{m}$ spectra reveal fainter components that are blueshifted from OMC-1 velocities and reach negative LSR velocities (see the spectra in Fig. 5). Goicoechea et al. (2015) already found that $\sim 15 \%$ of the [C II] $158 \mu \mathrm{m}$ luminosity toward the central regions of OMC-1 does not arise from its UV-illuminated surface. This $\mathrm{C}^{+}$emission mainly comes from the foreground half-shell that surrounds OMC-1 and expands (toward us) at $13 \mathrm{~km} \mathrm{~s}^{-1}$ (Pabst et al. 2019, 2020). The relatively narrow $[\mathrm{C}$ II $] 158 \mu \mathrm{m}$ line profiles $\left(\Delta v \approx 4 \mathrm{~km} \mathrm{~s}^{-1}\right)$ in the shell demonstrate that the gas is largely neutral. That is, dominated by $\mathrm{H}, \mathrm{H}_{2}$, and $\mathrm{C}^{+}$(with an ionization potential of $11.3 \mathrm{eV}$ ). Indeed, hydrogen recombination lines from fully ionized $\mathrm{H}$ II regions display much broader profiles $\left(\Delta v>15 \mathrm{~km} \mathrm{~s}^{-1}\right.$ for $T_{\mathrm{e}}>5000 \mathrm{~K}$; Churchwell et al. 1978). However, carbon recombination lines from the surface and edges of OMC-1, detected at radio (Natta et al. 1994; Salas et al. 2019) and millimeter wavelengths (Cuadrado et al. 2019), show narrow profiles $\Delta v=2.5-5 \mathrm{~km} \mathrm{~s}^{-1}$. These are typical of the neutral photodissociation region (PDR) that separates the hot H II gas from the cold molecular gas. The $8 \mu \mathrm{m}$ emission from UV-pumped PAHs also arises from PDR gas (e.g., Hollenbach \& Tielens 1997). Hence, the good correlation between the $\mathrm{C}^{+}$and PAH emission from the shell (Pabst et al. 2019), together with the narrow [C II] $158 \mu \mathrm{m}$ line-widths, supports the conclusion that most of the $\mathrm{C}^{+}$emission in the shell originates from neutral PDR gas rather than in the ionized gas.

\subsection{Detection of blue-shifted CO globules in the Veil}

The dotted rectangular box in Fig. 1 shows the specific area investigated in this work (expanded in Fig. 3). Despite the $0.75 \mathrm{~K} \mathrm{~km} \mathrm{~s}^{-1}(3 \sigma)$ sensitivity level of our ${ }^{12} \mathrm{CO}$ (2-1) map, equivalent to $N\left({ }^{12} \mathrm{CO}\right) \gtrsim(5-15) \times 10^{14} \mathrm{~cm}^{-2}$, we do not detect widespread and extended $\mathrm{CO}$ emission from the shell (i.e., blueshifted from the OMC). For the typical extinction $\left(A_{\mathrm{V}} \sim 1-\right.$ $2 \mathrm{mag})$ and plausible gas densities $\left(n_{\mathrm{H}}\right.$ of several $\left.10^{3} \mathrm{~cm}^{-3}\right)$ in this material (O'Dell 2001; Abel et al. 2016), UV photodissociation must severely restrict the formation of abundant $\mathrm{CO}$ (van Dishoeck \& Black 1988). Nevertheless, the lack of detectable extended $\mathrm{CO}$ emission does not directly imply that the whole shell is $100 \%$ atomic throughout and not molecular. 

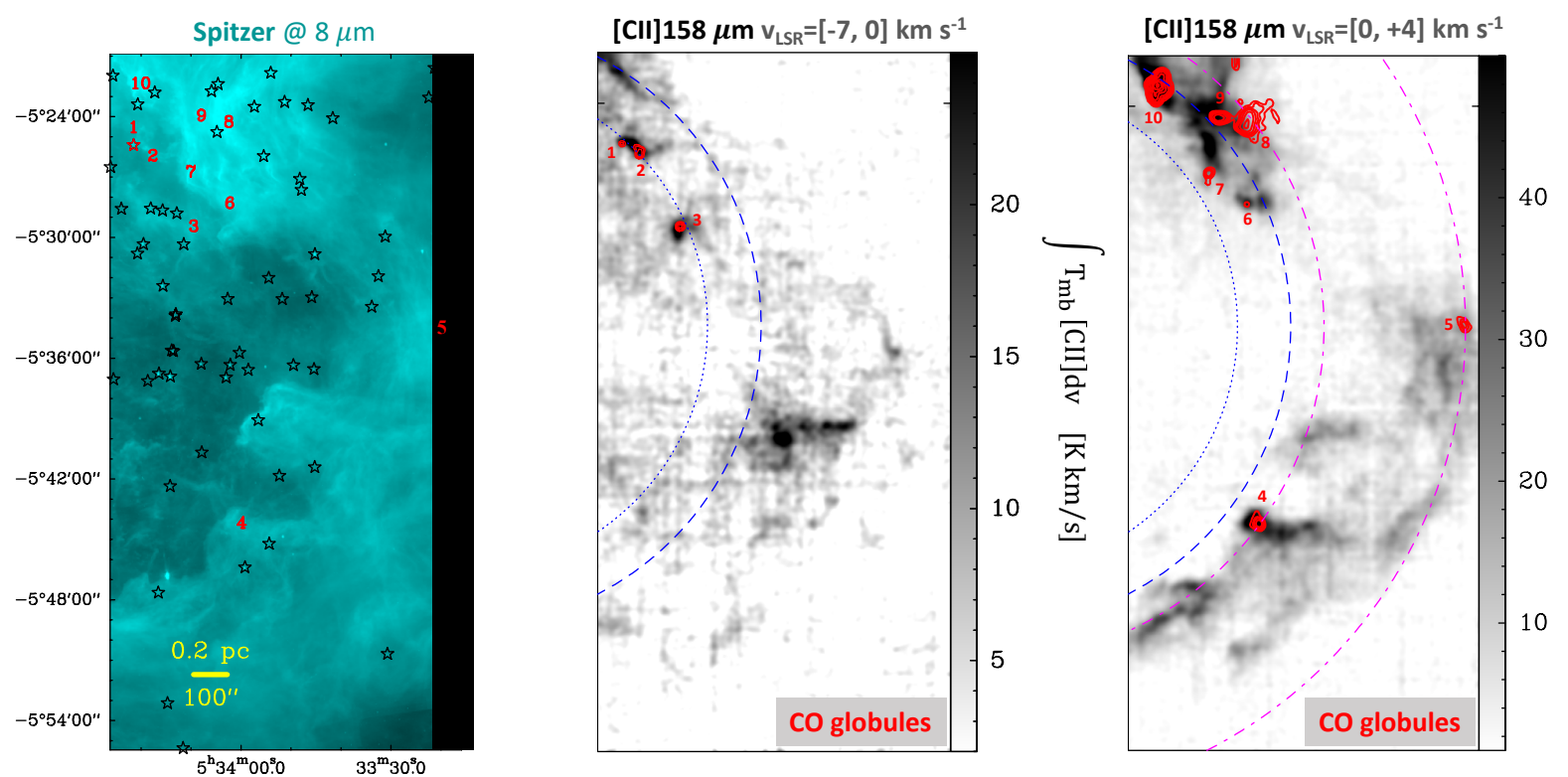

Fig. 4. CO globules in the shell that encloses the Veil bubble. Left panel: Spitzer's $8 \mu \mathrm{m}$ image (2" resolution). Numbers mark the position of the CO globules (shown in the middle and right panels). Stars show the position of YSOs detected in the field (Megeath et al. 2012, 2016). Globule \#1 matches the position of YSO \#1728 (red star). Middle panel: $[\mathrm{C}$ II $] 158 \mu \mathrm{m}$ emission at $v_{\mathrm{LSR}}=-7$ to $0 \mathrm{~km} \mathrm{~s}^{-1}$ (background grey image) and ${ }^{12} \mathrm{CO}$ globules \#1, \#2 and \#3 in the same velocity range (emission in red contours). Right panel: same as the middle panel but for the range $v_{\mathrm{LSR}}=0$ to $+4 \mathrm{~km} \mathrm{~s}^{-1}$. The arcs approximately represent the projection of concentric expanding rings in the shell (blue for rings closer to us).

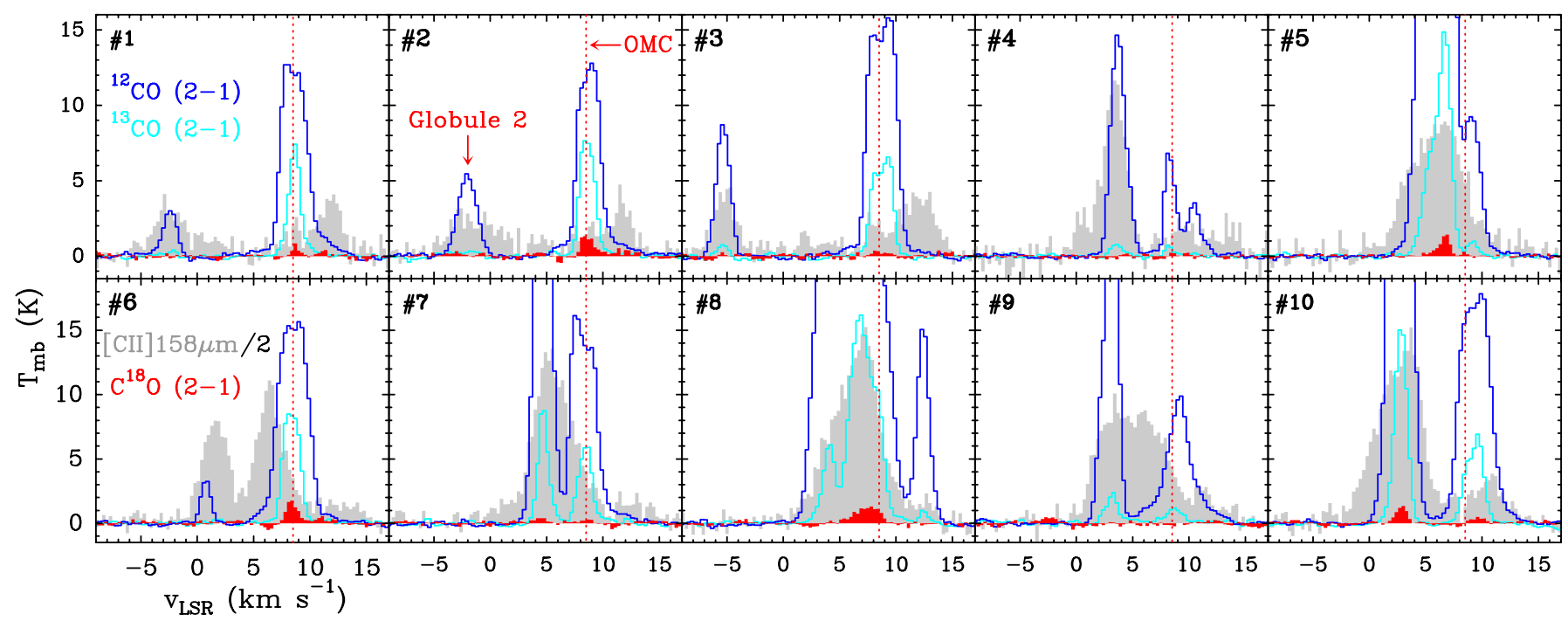

Fig. 5. Velocity-resolved spectra toward the $\mathrm{CO}$ globules in Orion's Veil. Each panel shows the $[\mathrm{C} \mathrm{II}] 158 \mu \mathrm{m}$ line (colored in gray), ${ }^{12} \mathrm{CO} J=2-1$ (dark blue), ${ }^{13} \mathrm{CO} J=2-1$ (cyan), and $\mathrm{C}^{18} \mathrm{O} J=2-1$ (red) lines toward the emission peak of each globule (labeled by symbol \# as in Figs. 3 and 4 ). The $x$-axis represents the LSR velocity in $\mathrm{km} \mathrm{s}^{-1}$. The vertical red dotted line marks the approximate velocity of the emission produced by OMC and associated star-forming molecular cloud located behind the Veil. All panels show emission features at velocities blueshifted from OMC. Globules $\# 1$, \#2, and \#3 represent the first detection of quiescent CO emission structures at negative LSR velocities in Orion.

Indeed, Fig. 4 shows the presence of globules and other $\mathrm{CO}$ emission structures blueshifted from the OMC. This includes the first detection of quiescent molecular gas at negative $v_{\mathrm{LSR}}$ (\#1, \#2, and \#3) and well separated from the main star-forming cores in the integral-shape filament (Johnstone \& Bally 1999; Kirk et al. 2017). We extracted the angular sizes of these globules using the native $\mathrm{CO}$ maps at $11^{\prime \prime}$ resolution. They range from $18^{\prime \prime}(\sim 7500 \mathrm{AU})$ to $80^{\prime \prime}(\sim 0.16 \mathrm{pc})$, with their fainter emission contour typically above $5 \sigma$.

Figure 6 shows a gallery with zooms on the globules and other blueshifted structures in the $\mathrm{CO}(2-1)$ (reddish) and $8 \mu \mathrm{m}$ band (bluish) emission. Figure A.1 shows the same gallery but displaying the [C $\mathrm{CI}] 158 \mu \mathrm{m}$ emission (bluish) integrated in exactly the same velocity range as the $\mathrm{CO}$ emission from each globule (i.e., $\mathrm{C}^{+}$that is strictly connected in velocity with CO). The smallest globules (\#1, \#2, \#3, and \#6) resemble the kind of tiny clouds originally seen in visible plates against the nebular emission from $\mathrm{H}$ II regions (ionized by radiation from nearby OB stars) and referred to as "globules" 2 Interestingly, no globule associated with the Orion nebula was found
in the original work of Bok \& Reilly (1947). 

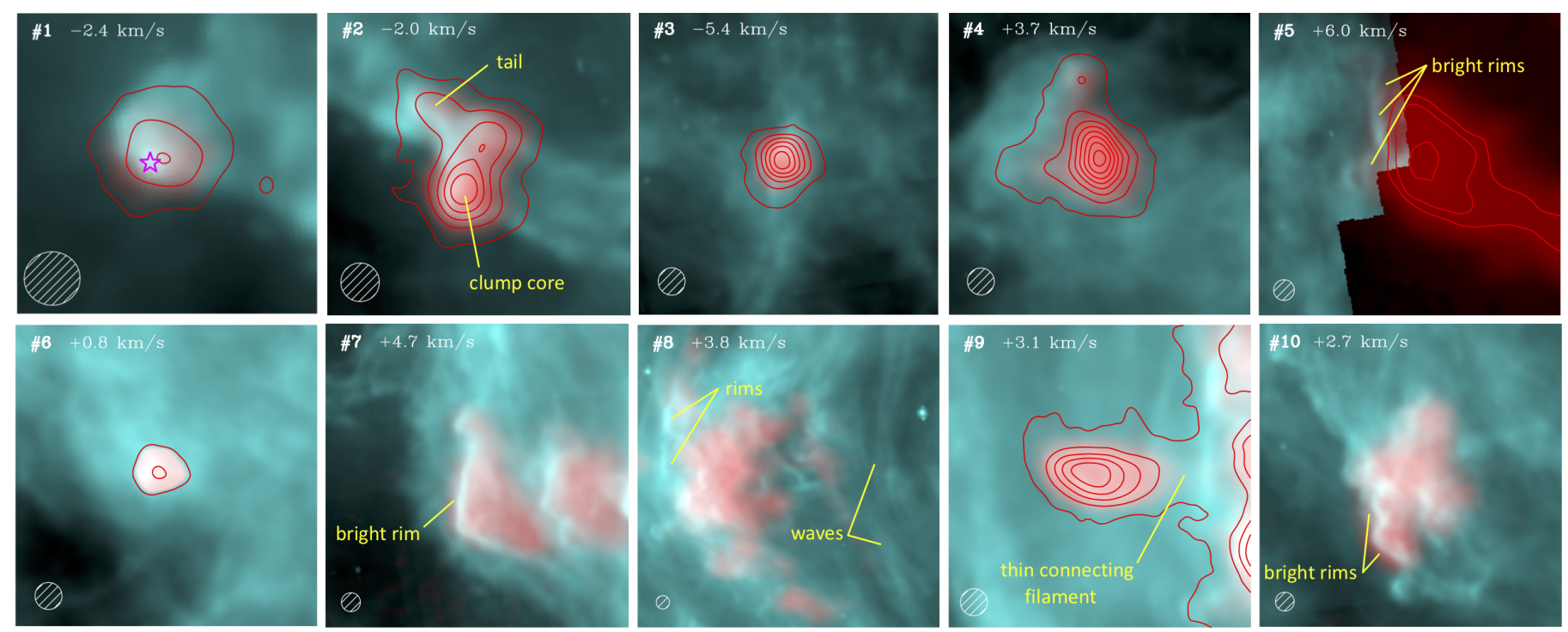

Fig. 6. Gallery of blueshifted $\mathrm{CO}$ globules and emission structures detected toward Orion's Veil bubble. The reddish color is the ${ }^{12} \mathrm{CO}(2-1)$ emission integrated over the appropriate emission velocity range of each globule (spectra shown in Fig. 5). The bluish color is the $8 \mu \mathrm{m}$ emission (extended PAH emission) imaged with Spitzer/IRAC at 2" resolution. Each panel indicates the LSR velocity centroid of the CO line-profiles and a white-lined circle with the $11^{\prime \prime}(\sim 4500 \mathrm{AU})$ beam size of the $\mathrm{CO}$ observations. The images of the smaller CO globules display ${ }^{12} \mathrm{CO}(2-1)$ intensity contours (in red) starting with the $5 \sigma \mathrm{rms}$ level (except for the brighter globules \#5 and \#9). In order to exhibit the bright rims (FUV-illuminated edges) of the larger and brighter $\mathrm{CO}$ globules, their images do not display $\mathrm{CO}$ emission contours (except for globule \#5, where the contours help to locate the head of a more elongated structure). Globule \#1 matches the position of YSO \#1728 (magenta star, Megeath et al. 2012). Figure A.1 shows the same gallery but displaying the velocity-resolved [C II] $158 \mu \mathrm{m}$ emission in bluish.

(Bok \& Reilly 1947; Minkowski 1949; Thackeray 1950), "cometary globules", or "tear drops" (Herbig 1974), and more recently "cusps" (De Marco et al. 2006) or "globulettes" when their sizes are smaller than about 10000 AU (Gahm et al. 2007). Hence, in this work we use the term "globule" in a generic sense to signify small and over-dense molecular gas blobs. Globules \#1, \#3, and \#6 show spherical morphologies but they are surrounded by extended $8 \mu \mathrm{m}$ (and [C II] $158 \mu \mathrm{m}$ ) emitting structures. Globules \#2 and \#4 are also roundish, but show indications of diffuse tails. These globules have $\mathrm{CO}$ velocity centroids that are significantly blueshifted from OMC and are isolated.

In addition, previous $\mathrm{CO}$ maps of OMC reveal more extended and peculiar structures (Shimajiri et al. 2011; Berné et al. 2014; Kong et al. 2018). Some of our blueshifted but positive- $v_{\text {LSR }}$ globules belong to these structures. They are located close to the surface of the dense molecular cloud (but are likely of a different nature) or at the limb-brightened edge of the shell. A very remarkable structure of this kind is the Kelvin-Helmholtz $(\mathrm{KH})$ "ripples" or "periodic undulations" studied by Berné et al. (2010) and indicated in our Figs. 2 and 3. Globule \#7 is the blueshifted head of the KH ripple, whereas \#5 in the westernmost part of the map is the tip of a more prominent and bright-rimmed structure, a pillar or "elephant trunk" that points toward the Trapezium. The extended structures associated with $\# 5$, \#8 and \#10 also show multiple far-UV (FUV; $E<13.6 \mathrm{eV}$ ) illuminated edges revealed by their bright $8 \mu \mathrm{m}$ rims delineating the $\mathrm{CO}$ emission. These globules must be facing strong FUV fluxes. Globule \#8 has a different morphology. It is part of a more extended region that is apparently connected to the $\mathrm{OMC}$, and is characterized by wavelike structures and $8 \mu \mathrm{m}$ rims pointing toward the Trapezium. The CO and [C II] $158 \mu \mathrm{m}$ emission follows an arched morphology also roughly pointing toward the Trapezium. Finally, globule \#9 appears to be detached from \#8, still showing a thin connecting filament.
The similar velocity centroid of the $\mathrm{CO}$ and [C II] $158 \mu \mathrm{m}$ lines toward each globule (Table A.2), as well as the spatial coincidence with velocity-coherent $\mathrm{C}^{+}$extended emission structures indicate that several globules are embedded in the shell (see position-velocity diagrams in Figs. 7 and A.2-A.9). Owing to the shell expansion, the negative- $v_{\mathrm{LSR}}$ globules should be located in the near side of the shell (closer to us) and not at the surface of the OMC. These globules resemble starless cores embedded in a $\mathrm{C}^{+}$-bright envelope (for globule \#4 with the morphology of the [C II] $158 \mu \mathrm{m}$ emission akin to a cometary globule, Fig. 4). Despite their relatively faint ${ }^{12} \mathrm{CO}$ emission levels, we also detect ${ }^{13} \mathrm{CO}$ toward most of them. However, with the exception of globules \#5 and \#10, we do not detect blueshifted $\mathrm{C}^{18} \mathrm{O}$ emission. The lack of $\mathrm{C}^{18} \mathrm{O}(2-1)$ emission is consistent with low extinction depths in the range of $A_{\mathrm{V}} \lesssim 3$ mag (Frerking et al. 1982; Cernicharo \& Guelin 1987; Pety et al. 2017).

To conclude this general presentation and analysis of the region, in Fig. 8 we show the approximate map of $G_{0}$, the stellar FUV flux in the line of sight toward the bubble $\left(G_{0} \simeq 1.7\right.$ is the mean radiation field in the solar neighborhood in Habing units; Habing 1968). These FUV photons heat grains, ionize C atoms, and dissociate $\mathrm{CO}$ molecules. Figure 8 shows that the innermost regions of the shell are directly exposed to FUV radiation from the Trapezium stars at $G_{0}$ levels of several hundred. The FUV flux reaching the outer portions of the shell is more attenuated, down to $G_{0} \sim 40$ (see Sect. 4.3 for details).

\subsection{Observed line parameters of the CO globules}

The ${ }^{12} \mathrm{CO}(2-1)$ line profiles are relatively narrow, with a median value of $\Delta v\left({ }^{12} \mathrm{CO}\right)=1.5 \mathrm{~km} \mathrm{~s}^{-1}$. These profiles are remarkably Gaussian (except for \#5 and \#8 that display blended components) and do not show the kind of line asymmetries or wings 


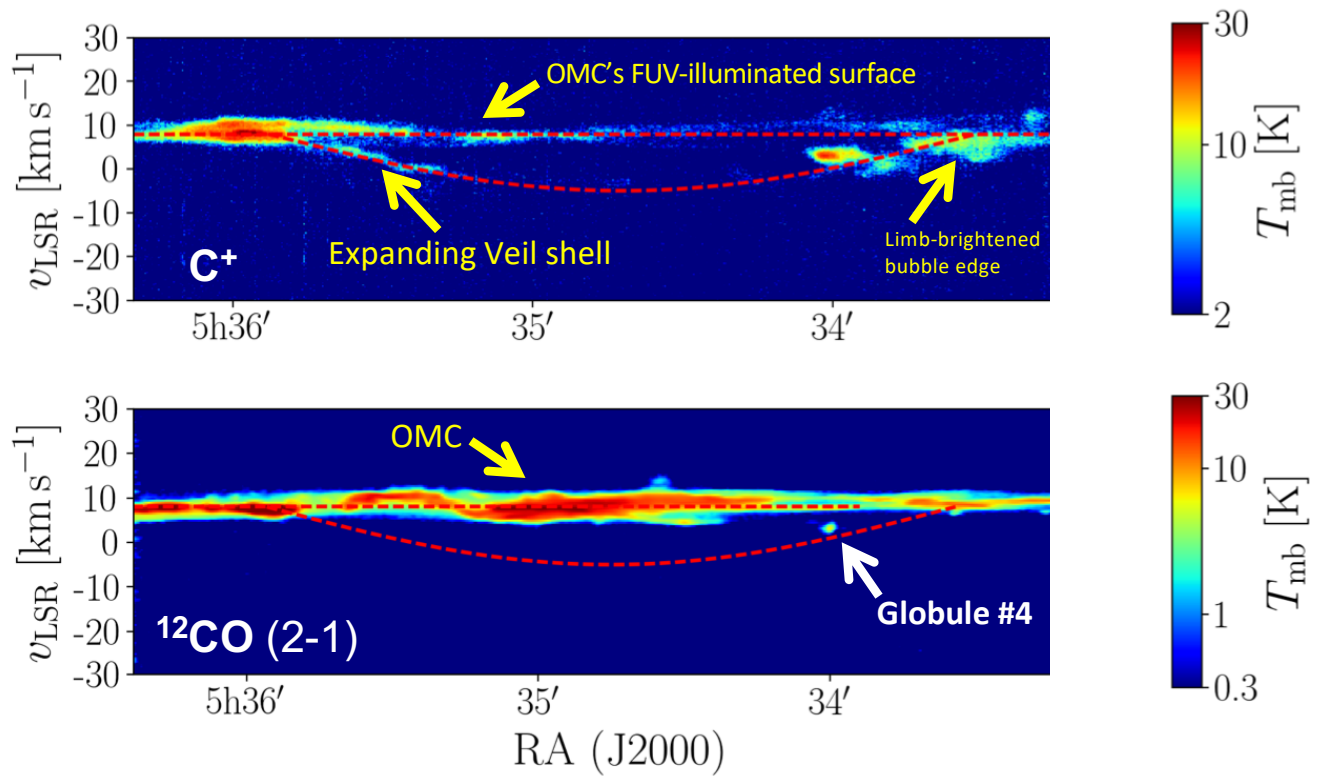

Fig. 7. Position-velocity diagram of the [C II] $158 \mu \mathrm{m}$ (upper panel) and $\mathrm{CO}$ (2-1) (lower panel) emission along an eastwest cut across the bubble at the declination of globule \#4 $\left(-5^{\circ} 44^{\prime} 11.55^{\prime \prime}\right)$. We created this diagram by averaging spectra over a cut of $45^{\prime \prime}$ wide in declination. A model of a half-shell expanding at $13 \mathrm{~km} \mathrm{~s}^{-1}$ is shown as a curved red dashed line (from Pabst et al. 2019). The bright emission at $v_{\mathrm{LSR}}=+8 \mathrm{~km} \mathrm{~s}^{-1}$ (horizontal red dashed line) corresponds to the background dense molecular cloud and the integral-shape filament.

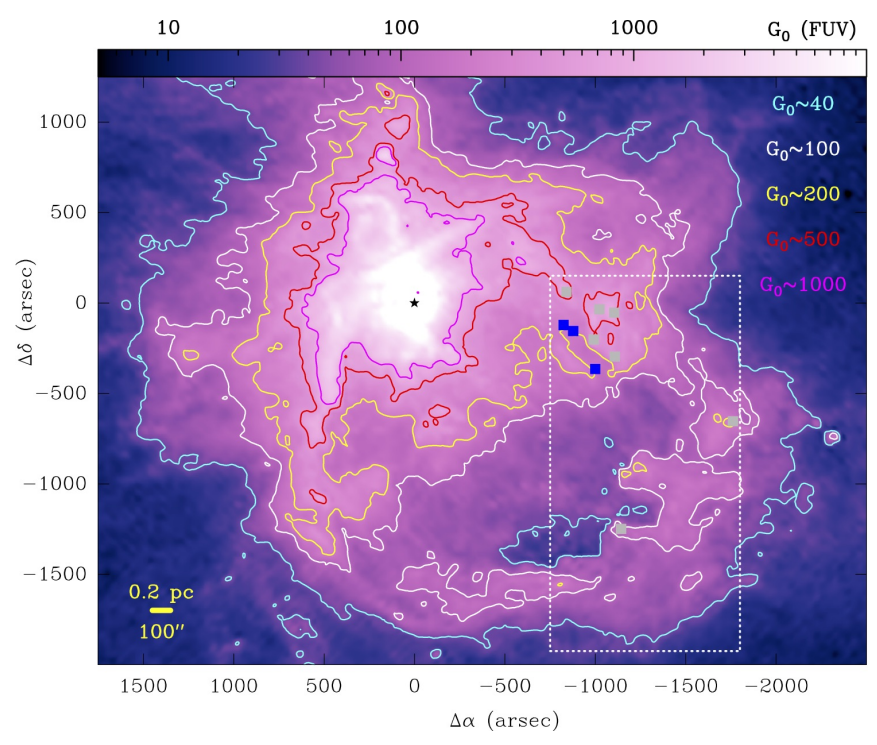

Fig. 8. One-square-degree image of the flux of nonionizing FUV photons $\left(G_{0}\right.$ in units of the Habing field) along lines of sight toward the Veil bubble. The black star at $\left(0^{\prime \prime}, 0^{\prime \prime}\right)$ corresponds to the position of the illuminating star $\theta^{1}$ Ori $C$. Here, we focus on the area enclosed by the dotted box. The blue and gray squares mark the position of the detected $\mathrm{CO}$ globules (blue squares for the negative- $v_{\text {LSR }}$ globules).

expected in collapsing, or expanding, or outgassing globules. The velocity centroid of both ${ }^{12} \mathrm{CO}$ and ${ }^{13} \mathrm{CO}$ lines coincides for each globule (both species arise from the same gas component). However, the ${ }^{13} \mathrm{CO}(2-1)$ lines are narrower, that is, $\Delta v\left({ }^{13} \mathrm{CO}\right)=$ $1.2 \mathrm{~km} \mathrm{~s}^{-1}$ (median), indicating that the ${ }^{12} \mathrm{CO}(2-1)$ lines are opacity-broadened (Phillips et al. 1979). The [C II] $158 \mu \mathrm{m}$ lines toward the globules are significantly broader, that is, $\Delta v\left(\mathrm{C}^{+}\right)=3.2 \mathrm{~km} \mathrm{~s}^{-1}$ (median), and show more intricate line profiles. Assuming $T_{\mathrm{k}}=100 \mathrm{~K}$ for the $\mathrm{C}^{+}$gas (Pabst et al. 2019), $T_{\mathrm{k}}=10 \mathrm{~K}$ for ${ }^{13} \mathrm{CO}$, and separating the thermal broadening $\left(\Delta \sigma_{\text {th }}\left(\mathrm{C}^{+}\right)=0.26 \mathrm{~km} \mathrm{~s}^{-1}\right.$ and $\left.\Delta \sigma_{\text {th }}\left({ }^{13} \mathrm{CO}\right)=0.05 \mathrm{~km} \mathrm{~s}^{-1}\right)$, the differences in observed line widths imply that the [C II] $158 \mu \mathrm{m}$ emission arises from a more turbulent gas that surrounds the ${ }^{13} \mathrm{CO}$ cores, with $\sigma_{\text {turb }}\left(\mathrm{C}^{+}\right) / \sigma_{\text {turb }}\left({ }^{13} \mathrm{CO}\right) \simeq 1.3 / 0.5$
$\left(\Delta v_{\mathrm{obs}}=2.35 \sigma_{\mathrm{obs}}\right.$ and $\left.\sigma_{\mathrm{obs}}^{2}=\sigma_{\mathrm{turb}}^{2}+\sigma_{\mathrm{th}}^{2}\right)$. These dispersions imply transonic to supersonic motions.

\section{Analysis}

Wide-field near-IR and visible images of $\mathrm{H}$ II regions allow the detection of globules of neutral gas and dust in their surroundings (e.g., De Marco et al. 2006; Gahm et al. 2007). Detected in silhouette or as bright cusps, photometric images reveal the morphology and projected position of these compact objects. Velocity-resolved $\mathrm{C}^{+}$and $\mathrm{CO}$ spectroscopic images (at sub-km s${ }^{-1}$ resolution) help to find these globules in velocity space, and also provide a means of quantifying their physical conditions, bulk masses, and gas kinematics.

A few studies of this kind have previously focused on small $\mathrm{CO}$ globules seen in planetary nebulae around evolved stars such as the Helix Nebula (e.g., Huggins et al. 1992, 2002) and on CO globules seen toward H II regions in more distant high-mass starforming regions such as the Rosette Nebula (Schneps et al. 1980; Gonzalez-Alfonso \& Cernicharo 1994; Dent et al. 2009; Gahm et al. 2013) located at a distance of $1.6 \mathrm{kpc}$. In this section, we analyze the [C II $] 158 \mu \mathrm{m},{ }^{12} \mathrm{CO}$, and ${ }^{13} \mathrm{CO}$ emission from the globules detected in Orion at high spatial resolution.

\subsection{Single-slab analysis}

With the detection of a single rotational line, and given the possible small-scale structure and potential gradients in the physical conditions of these globules, the derivation of their gas density, temperature, and mass is not trivial. In this section, we assume that the $\mathrm{CO}$ level populations are characterized by a single excitation temperature (i.e., single-slab analysis). Given the low critical densities for collisional excitation of the observed CO lines $\left(n_{\mathrm{cr}, 2-1} \approx 10^{4} \mathrm{~cm}^{-3}\right)$, we implicitly assume that the ${ }^{12} \mathrm{CO}$ levels are close to thermalization $\left(T_{\mathrm{ex}} \simeq T_{\mathrm{k}}\right)$ and that ${ }^{13} \mathrm{CO}$ is a good tracer of the total molecular column density. This is obviously a first-order approach (e.g., at low densities the higher- $J$ lines will be subthermally excited) but it is highly complementary to the mere photometric detection of these globules. In Sect. 4.4 we perform a more detailed depth-dependent modeling. 
A\&A 639, A1 (2020)

Table 1. Globule parameters estimated from line observations adopting a reasonable range of ${ }^{12} \mathrm{CO}$ abundances (single-slab approximation).

\begin{tabular}{|c|c|c|c|c|c|c|c|}
\hline Globule & $\begin{array}{l}\text { Coordinates } \\
\text { (RA Dec) }\end{array}$ & $\begin{array}{c}R_{\mathrm{g}} \\
(\mathrm{AU}) \\
\end{array}$ & $\begin{array}{c}T_{\text {ex }}(\mathrm{CO})^{(a)} \\
(\mathrm{K}) \\
\end{array}$ & $\begin{array}{l}N(\mathrm{CO})^{(b)} \\
\left(\mathrm{cm}^{-2}\right) \\
\end{array}$ & $\begin{array}{c}x(\mathrm{CO}) \\
\left(10^{-5} \text { adopted }\right) \\
\end{array}$ & $\begin{array}{c}n_{\mathrm{H}}{ }^{(c)} \\
\left(10^{4} \mathrm{~cm}^{-3}\right) \\
\end{array}$ & $\begin{array}{l}A_{\mathrm{V}}{ }^{(d)} \\
(\mathrm{mag})\end{array}$ \\
\hline$\# 1$ & $5 \mathrm{~h} 34 \mathrm{~m} 21.21 \mathrm{~s}-5^{\circ} 25^{\prime} 24.23^{\prime \prime}$ & 3700 & $7.6 \pm 0.6$ & $(3.8 \pm 0.5) \times 10^{16}$ & $1.5-0.5$ & $4.8 \pm 2.8$ & $1.9 \pm 1.1$ \\
\hline \#2 & $5 \mathrm{~h} 34 \mathrm{~m} 17.65 \mathrm{~s}-5^{\circ} 25^{\prime} 57.52^{\prime \prime}$ & 7000 & $10.0 \pm 0.9$ & (same as \#1) & $1.5-0.5$ & $2.5 \pm 1.5$ & $1.9 \pm 1.1$ \\
\hline \#3 & $5 \mathrm{~h} 34 \mathrm{~m} 09.55 \mathrm{~s}-5^{\circ} 29^{\prime} 29.40^{\prime \prime}$ & 5800 & $13.8 \pm 1.4$ & $(3.8 \pm 0.2) \times 10^{16}$ & $1.5-0.5$ & $3.0 \pm 1.6$ & $1.8 \pm 1.0$ \\
\hline \#4 & $5 \mathrm{~h} 33 \mathrm{~m} 59.86 \mathrm{~s}-5^{\circ} 44^{\prime} 11.55^{\prime \prime}$ & 8300 & $19.8 \pm 2.2$ & $(3.8 \pm 0.1) \times 10^{16}$ & $1.5-0.5$ & $2.1 \pm 1.1$ & $1.8 \pm 0.9$ \\
\hline \#5 & $5 \mathrm{~h} 33 \mathrm{~m} 18.44 \mathrm{~s}-5^{\circ} 34^{\prime} 16.29^{\prime \prime}$ & 7500 & $46.2 \pm 6.0$ & $(1.8 \pm 0.2) \times 10^{18}$ & $15.0-5.0$ & $10.9 \pm 6.1$ & $8.5 \pm 4.8$ \\
\hline \#6 & $5 \mathrm{~h} 34 \mathrm{~m} 02.27 \mathrm{~s}-5^{\circ} 28^{\prime} 18.58^{\prime \prime}$ & 3100 & $7.9 \pm 0.6$ & $(2.8 \pm 0.3) \times 10^{16}$ & $1.5-0.5$ & $4.3 \pm 2.5$ & $1.4 \pm 0.8$ \\
\hline \#7 & $5 \mathrm{~h} 34 \mathrm{~m} 10.00 \mathrm{~s}-5^{\circ} 26^{\prime} 44.24^{\prime \prime}$ & 6200 & $46.4 \pm 6.1$ & $(5.8 \pm 0.5) \times 10^{17}$ & $15.0-5.0$ & $4.3 \pm 2.4$ & $2.8 \pm 1.6$ \\
\hline \#8 & $5 \mathrm{~h} 34 \mathrm{~m} 02.46 \mathrm{~s}-5^{\circ} 24^{\prime} 17.04^{\prime \prime}$ & 16600 & $34.4 \pm 4.4$ & $(3.0 \pm 0.3) \times 10^{17}$ & $15.0-5.0$ & $0.9 \pm 0.5$ & $1.5 \pm 0.8$ \\
\hline \#9 & $5 \mathrm{~h} 34 \mathrm{~m} 07.99 \mathrm{~s}-5^{\circ} 23^{\prime} 57.64^{\prime \prime}$ & 7200 & $31.8 \pm 4.0$ & $(1.4 \pm 0.1) \times 10^{17}$ & $15.0-5.0$ & $0.9 \pm 0.5$ & $0.7 \pm 0.4$ \\
\hline \multirow[t]{2}{*}{$\# 10$} & $5 \mathrm{~h} 34 \mathrm{~m} 20.14 \mathrm{~s}-5^{\circ} 22^{\prime} 21.93^{\prime \prime}$ & 14500 & $58.0 \pm 7.9$ & $(1.5 \pm 0.2) \times 10^{18}$ & $15.0-5.0$ & $4.7 \pm 2.7$ & $7.6 \pm 4.6$ \\
\hline & Median & 7100 & 25.8 & $8.6 \times 10^{16}$ & $8.3-2.8$ & 3.6 & 1.8 \\
\hline
\end{tabular}

Notes. ${ }^{(a)}$ Assuming optically thick ${ }^{12} \mathrm{CO}$ emission and taking into account absolute intensity calibration errors and $1 \sigma$ errors discussed in Sect. 2. ${ }^{(b)}$ From optically thin ${ }^{13} \mathrm{CO}$ emission. ${ }^{(c)}$ For $n_{\mathrm{H}}=N_{\mathrm{H}} / 2 R_{\mathrm{g}}$ with $N_{\mathrm{H}}=N(\mathrm{CO}) / x(\mathrm{CO})_{\text {adopted }}{ }^{\left({ }^{(d)}\right.}$ Defined as $A_{\mathrm{V}}=3.5 \times 10^{-22} N\left({ }^{12} \mathrm{CO}\right) / x\left({ }^{12} \mathrm{CO}\right)_{\text {adopted }}$.

The observed $W\left({ }^{12} \mathrm{CO}\right) / W\left({ }^{13} \mathrm{CO}\right)$ integrated line intensity ratio toward all globules is always lower than 25 (with $W=$ $\int T_{\mathrm{mb}} \mathrm{dv}$ in $\mathrm{K} \mathrm{km} \mathrm{s}^{-1}$; see Fig. A.10). This is lower than the ${ }^{12} \mathrm{C} /{ }^{13} \mathrm{C}$ isotopic ratio $\left(R_{12 \mathrm{C} 13}\right)$ in OMC-1 $\left(R_{12 \mathrm{C} 13}=67 \pm 3\right.$; Langer \& Penzias 1990) and implies that: (i) the ${ }^{12} \mathrm{CO}$ emission is optically thick, (ii) the ${ }^{13} \mathrm{CO}$ abundance is enhanced over the expected isotopic ratio, so-called chemical fractionation (Langer et al. 1984), or (iii) both. For optically thick lines, the ${ }^{12} \mathrm{CO}(2-1)$ excitation temperature $T_{\mathrm{ex}}$ is a good lower limit on $T_{\mathrm{k}}$, and we can directly extract this from the line peak temperature, $T_{\mathrm{P}, 12}$, of each globule (see Table A.1):

$T_{\mathrm{ex}}=\frac{h v / k}{\ln \left(1+\frac{h v / k}{T_{\mathrm{P}, 12}+J\left(T_{\mathrm{bg}}\right)}\right)}$,

where $J(T)=(h v / k) /\left(e^{h v / k T}-1\right)$ is the equivalent brightness temperature of a black body at $T$, and $T_{\mathrm{bg}}$ is the cosmic background temperature $2.7 \mathrm{~K}$. We note that we spatially resolve these globules, and so no beam-filling factor correction is needed. Taking into account that $T_{\text {ex }}\left({ }^{13} \mathrm{CO} 2-1\right)=f \times T_{\text {ex }}\left({ }^{12} \mathrm{CO} 2-1\right)$, where $f \leq 1$, we can determine the opacity of the ${ }^{13} \mathrm{CO}(2-1)$ line, $\tau_{13}=-\ln \left(1-\frac{T_{\mathrm{P}, 13}}{J\left(T_{\mathrm{ex}}\right)-J\left(T_{\mathrm{bg}}\right)}\right)$, from observations, and also the ${ }^{13} \mathrm{CO}$ column density:

$N\left({ }^{13} \mathrm{CO}\right)=N_{\text {thin }}\left({ }^{13} \mathrm{CO}\right) \frac{\tau_{13}}{1-e^{-\tau_{13}}}$,

where $N_{\text {thin }}$ is the ${ }^{13} \mathrm{CO}$ column density (in $\mathrm{cm}^{-2}$ ) in the $\tau_{13} \rightarrow 0$ limit (see Eq. (3)). The factor $f$ reflects the possible different excitation temperatures of ${ }^{12} \mathrm{CO}(2-1)$ and ${ }^{13} \mathrm{CO}(2-1)$ lines due to line-trapping effects as the ${ }^{12} \mathrm{CO}(2-1)$ line opacity increases. The parameter $f$ tends to 1 in collisionally excited optically thin gas. We carried out nonlocal thermodynamic equilibrium (NLTE) calculations that show that line-trapping reduces $f$ for optically thick ${ }^{12} \mathrm{CO}$ emission (roughly above $N\left({ }^{12} \mathrm{CO}\right.$ ) of a few $10^{16} \mathrm{~cm}^{-2}$ ) and at low $n\left(\mathrm{H}_{2}\right)$ densities (typically lower than $\sim 10^{4} \mathrm{~cm}^{-3}$, the critical density of the $J=2-1$ transition). For the expected gas densities and $N(\mathrm{CO})$ in these globules, we find $f \simeq 0.9$ and this is the factor we use here. We calculate $N\left({ }^{13} \mathrm{CO}\right)$ assuming a Boltzmann distribution of the level populations at a uniform $T_{\mathrm{ex}}$. In this case,

$N_{\text {thin }}=8 \pi\left(\frac{v}{c}\right)^{3} \frac{Q\left(T_{\mathrm{ex}}\right)}{g_{2} A_{21}} \frac{e^{E_{2} / k T_{\mathrm{ex}}}}{e^{h v / k T_{\mathrm{ex}}}-1} \frac{W}{J\left(T_{\mathrm{ex}}\right)-J\left(T_{\mathrm{bg}}\right)}$,

where $W$ is the ${ }^{13} \mathrm{CO}(2-1)$ line integrated intensity for each globule (values tabulated in Table A.2).

We derive the ${ }^{12} \mathrm{CO}$ column density as $N\left({ }^{12} \mathrm{CO}\right)=R_{12 \mathrm{C} 13}$. $N\left({ }^{13} \mathrm{CO}\right)$, and the column density of gas across each $\mathrm{CO}$ globule core, $N_{\mathrm{H}}=N(\mathrm{H})+2 N\left(\mathrm{H}_{2}\right)$, defined as $N_{\mathrm{H}}=N\left({ }^{12} \mathrm{CO}\right) / x\left({ }^{12} \mathrm{CO}\right)$. Supported by our more detailed photochemical modeling (following section), we estimate the extinction $\left(A_{\mathrm{V}}\right)$ through each globule and their mass $\left(M_{\mathrm{g}}\right)$ adopting a plausible range of (uniform) $\mathrm{CO}$ abundances. We use $x\left({ }^{12} \mathrm{CO}\right)=(0.5-1.5) \times 10^{-5}$ when $N\left({ }^{12} \mathrm{CO}\right)<5 \times 10^{16} \mathrm{~cm}^{-2}$ (the most translucent case) and $x\left({ }^{12} \mathrm{CO}\right)=(0.5-1.5) \times 10^{-4}$ otherwise.

In order to determine $M_{\mathrm{g}}$, we use the observed angular sizes of each globule. Table 1 summarizes the radius $\left(R_{\mathrm{g}}\right)$ of each globule, and the range of gas densities $\left(n_{\mathrm{H}} \simeq N_{\mathrm{H}} / 2 R_{\mathrm{g}}\right)$ and molecular core depths (defined as $A_{\mathrm{V}}=3.5 \times 10^{-22} N\left({ }^{12} \mathrm{CO}\right) / x\left({ }^{12} \mathrm{CO}\right.$ ), see Sect. 4.4) derived in the single-slab approximation.

In this approach, the most critical error parameter in the calculated values is the adopted range of $\mathrm{CO}$ abundances. The adopted $x\left({ }^{12} \mathrm{CO}\right)$ range (within a factor of three) roughly agrees with specific PDR models adapted to the UV illuminating conditions in the shell (see Sect. 4.4). The resulting range of calculated $A_{\mathrm{V}}$ values in each globule brackets the extinction values we obtain from the more detailed PDR models as well. They are also consistent with the nondetection of $\mathrm{C}^{18} \mathrm{O}$ emission, which approximately implies $A_{\mathrm{V}}<3 \mathrm{mag}$ for all globules (except for the globules \#5 and \#10).

Taking into account the achieved rms sensitivity of our maps, we also computed the minimum beam-averaged ${ }^{12} \mathrm{CO}$ column density, $N_{\min }\left({ }^{12} \mathrm{CO}\right)$, that we could have detected. We adopt $W_{3 \sigma}=3 \sigma \sqrt{2 \delta \mathrm{v} \Delta v}$ with $\sigma=0.25 \mathrm{~K}$ (the rms noise level of our native map), $\delta v=0.25 \mathrm{~km} \mathrm{~s}^{-1}$ (the velocity channel resolution), $\Delta v=2 \mathrm{~km} \mathrm{~s}^{-1}$ (the expected line-width). This leads to $W_{3 \sigma}=0.75 \mathrm{~K} \mathrm{~km} \mathrm{~s}^{-1}$. For $T_{\mathrm{ex}}=5-20 \mathrm{~K}$, our map is sensitive to a beam-averaged $N_{\min }\left({ }^{12} \mathrm{CO}\right)>(5-15) \times 10^{14} \mathrm{~cm}^{-2}(3 \sigma)$. Assuming a typical shell thickness of $1.8 \mathrm{mag}$ of visual extinction in the Veil (O'Dell 2001), this limit is equivalent to detecting 
Table 2. Velocity dispersions, pressures, and relevant masses of each globule molecular core (from ${ }^{12} \mathrm{CO}$ and ${ }^{13} \mathrm{CO}$ ) and their envelopes $\left(\right.$ from $\mathrm{C}^{+}$).

\begin{tabular}{rccccccccc}
\hline \hline Globule & $\begin{array}{c}\sigma_{\mathrm{nth}}\left(\mathrm{C}^{+}\right) \\
\left(\mathrm{km} \mathrm{s}^{-1}\right)^{(a)}\end{array}$ & $\begin{array}{c}P_{\mathrm{nth}} / k\left(\mathrm{C}^{+}\right)^{(a)} \\
\left(10^{6} \mathrm{~cm}^{-3} \mathrm{~K}\right)\end{array}$ & $\begin{array}{c}P_{\mathrm{th}} / k\left(\mathrm{C}^{+}\right)^{(a)} \\
\left(10^{6} \mathrm{~cm}^{-3} \mathrm{~K}\right)\end{array}$ & $\begin{array}{c}\sigma_{\mathrm{nth,}}{ }^{(b)} \\
\left(\mathrm{km} \mathrm{s}^{-1}\right)\end{array}$ & $\begin{array}{c}P_{\mathrm{nth}, \mathrm{g}} / k^{(b)} \\
\left(10^{6} \mathrm{~cm}^{-3} \mathrm{~K}\right)\end{array}$ & $\begin{array}{c}P_{\mathrm{th}, \mathrm{g}} / k\left(T_{\mathrm{ex}}\right)^{(c)} \\
\left(10^{6} \mathrm{~cm}^{-3} \mathrm{~K}\right)\end{array}$ & $\begin{array}{c}M_{\mathrm{g}} \\
\left(M_{\odot}\right)\end{array}$ & $\begin{array}{c}m_{\mathrm{BE}} \\
\left(M_{\odot}\right)^{(c, d)}\end{array}$ & $\begin{array}{c}M_{\mathrm{J}} \\
\left(M_{\odot}\right)^{(e)}\end{array}$ \\
\hline$\# 1$ & $2.3(0.3)$ & $42.2 \pm 24.5$ & $4.8 \pm 2.8$ & $0.5(0.2)$ & $3.8 \pm 2.2$ & $0.4 \pm 0.2$ & $0.08 \pm 0.05$ & $0.03 \pm 0.01$ & $3.0(2.4)$ \\
$\# 2$ & $2.8(0.2)$ & $33.0 \pm 19.8$ & $2.5 \pm 1.5$ & $0.5(0.1)$ & $1.8 \pm 1.1$ & $0.3 \pm 0.2$ & $0.3 \pm 0.2$ & $0.06 \pm 0.03$ & $5.1(3.4)$ \\
$\# 3$ & $1.0(0.1)$ & $5.3 \pm 2.8$ & $3.0 \pm 1.6$ & $0.5(0.1)$ & $1.8 \pm 1.0$ & $0.5 \pm 0.3$ & $0.2 \pm 0.1$ & $0.2 \pm 0.1$ & $3.6(0.6)$ \\
$\# 4$ & $1.0(0.1)$ & $3.5 \pm 1.8$ & $2.1 \pm 1.1$ & $0.5(0.1)$ & $1.5 \pm 0.8$ & $0.5 \pm 0.3$ & $0.4 \pm 0.2$ & $0.6 \pm 0.3$ & $6.3(1.0)$ \\
$\# 5$ & $2.5(0.1)$ & $117.4 \pm 65.6$ & $10.9 \pm 6.1$ & $0.9(0.1)$ & $22.1 \pm 12.4$ & $5.4 \pm 3.5$ & $1.5 \pm 0.8$ & $0.7 \pm 0.4$ & $15.5(1.5)$ \\
$\# 6$ & $1.1(0.1)$ & $9.4 \pm 5.4$ & $4.3 \pm 2.5$ & $0.5(0.2)$ & $3.3 \pm 1.9$ & $0.4 \pm 0.3$ & $0.05 \pm 0.03$ & $0.06 \pm 0.03$ & $2.4(1.2)$ \\
$\# 7$ & $1.5(0.1)$ & $15.6 \pm 8.7$ & $4.3 \pm 2.4$ & $0.4(0.1)$ & $2.4 \pm 1.4$ & $2.1 \pm 1.4$ & $0.3 \pm 0.2$ & $1.8 \pm 0.9$ & $3.7(2.0)$ \\
$\# 8$ & $1.2(0.2)$ & $2.2 \pm 1.2$ & $0.9 \pm 0.5$ & $0.4(0.1)$ & $0.4 \pm 0.2$ & $0.3 \pm 0.2$ & $1.2 \pm 0.7$ & $2.5 \pm 1.2$ & $8.4(5.1)$ \\
$\# 9$ & $1.0(0.1)$ & $1.6 \pm 0.9$ & $0.9 \pm 0.5$ & $0.6(0.1)$ & $0.8 \pm 0.4$ & $0.3 \pm 0.2$ & $0.11 \pm 0.06$ & $2.4 \pm 1.2$ & $7.0(1.0)$ \\
$\# 10$ & $2.0(0.1)$ & $33.4 \pm 19.2$ & $4.7 \pm 2.7$ & $0.5(0.1)$ & $3.7 \pm 2.1$ & $3.0 \pm 2.0$ & $4.7 \pm 2.7$ & $2.1 \pm 1.1$ & $12.3(1.9)$ \\
\hline Median & 1.3 & 12.5 & 3.6 & 0.5 & 2.1 & 0.4 & 0.3 & 0.6 & 5.6 \\
\hline
\end{tabular}

Notes. Based on the range of globule densities estimated in Table 1 . Values in parenthesis are $1 \sigma$ errors. ${ }^{(a)}$ Assuming $T_{\mathrm{k}}=100 \mathrm{~K} .{ }^{\left({ }^{b}\right)}$ From ${ }^{13} \mathrm{CO}(2-1)$ line-widths. ${ }^{(c)}$ Assuming $T_{\mathrm{k}}=T_{\mathrm{ex}}\left({ }^{12} \mathrm{CO}\right) .{ }^{(d)}$ Bonnor-Ebert mass for an external pressure given by $P_{\mathrm{ext}}=P_{\mathrm{nth}}\left(\mathrm{C}^{+}\right)+P_{\mathrm{th}}\left(\mathrm{C}^{+}\right) .{ }^{(e)} \mathrm{Jeans}$ mass for $R_{\mathrm{g}}$ and $\sigma_{\mathrm{nth}+\mathrm{th}, \mathrm{g}}$ determined from ${ }^{13} \mathrm{CO}(2-1)$.

$x\left({ }^{12} \mathrm{CO}\right)$ abundances above $(1-3) \times 10^{-7}$ in the shell. This threshold seems high, but we highlight the strong UV illumination conditions and low extinction depth of this foreground component (i.e., low molecular column densities). On the other hand, the $N\left({ }^{12} \mathrm{CO}\right)$ column we detect toward the negative- $v_{\mathrm{LSR}}$ globules is at least 25 times higher than $N_{\min }\left({ }^{12} \mathrm{CO}\right)$. This implies that widespread and abundant $\mathrm{CO}$ is largely absent from the shell.

\subsection{Globule velocity dispersions, pressures, and masses}

Table 2 summarizes the properties of the interior of each globule (as traced by ${ }^{13} \mathrm{CO}$ ) and of their envelope and/or surroundings (as traced by $\mathrm{C}^{+}$). This table displays the nonthermal (turbulent) and thermal gas pressures, the estimated Bonnor-Ebert mass for a pressure-confined isothermal sphere $\left(m_{\mathrm{BE}}\right.$; Ebert 1955; Bonnor 1956), and the Jeans mass ( $M_{\mathrm{J}}$; e.g., Larson 1978). To derive these masses, we assume $T_{\mathrm{k}}=T_{\mathrm{ex}}\left({ }^{12} \mathrm{CO} 2-1\right)$ and use:

$m_{\mathrm{BE}}=1.15\left(\frac{c_{\mathrm{s}}}{0.2 \mathrm{~km} \mathrm{~s}^{-1}}\right)^{4}\left(\frac{P_{\mathrm{ext}}}{10^{5} \mathrm{~cm}^{-3} \mathrm{~K}}\right)^{-0.5}$,

from Lada et al. (2008), where $c_{\mathrm{S}}$ is the temperature-dependent speed of sound inside the globule. For the Jeans mass we use:

$M_{\mathrm{J}}=\frac{5 R_{\mathrm{g}} \sigma^{2}}{2 G}$,

from Kirk et al. (2017), where $\sigma$ includes the dominant nonthermal support inside each globule (from ${ }^{13} \mathrm{CO}$ line-widths). The resulting median values of the sample are $M_{\mathrm{g}}=0.3 M_{\odot}$, $m_{\mathrm{BE}}=0.6 M_{\odot}$, and $M_{\mathrm{J}}=5.6 M_{\odot}$ (see Table 2 individually).

\subsection{Stellar FUV photon flux $\left(G_{0}\right)$ toward the shell}

In Goicoechea et al. $(2015,2019)$ we estimated $G_{0}$ in OMC-1 from the integrated far-IR (FIR) dust thermal emission observed by Herschel. When dust grains absorb FUV photons, they are heated up, and re-radiate at FIR wavelengths. For a face-on PDR:

$G_{0} \simeq \frac{1}{2} \frac{I_{\mathrm{FIR}}\left(\mathrm{erg} \mathrm{s}^{-1} \mathrm{~cm}^{-2} \mathrm{sr}^{-1}\right)}{1.3 \times 10^{-4}}$ from Hollenbach \& Tielens (1997), where $G_{0}$ is the FUV radiation field in Habing units $\left(1.6 \times 10^{-3} \mathrm{erg} \mathrm{s}^{-1} \mathrm{~cm}^{-2}\right.$; Habing 1968), and $T_{\mathrm{d}, \mathrm{PDR}} \simeq 12.2 G_{0}^{0.2}$ is a characteristic dust temperature in the PDR (Hollenbach et al. 1991). In practice, the longer wavelength submillimeter (submm) dust emission toward the lines-of-sight of large column density may not only be produced by FUVheated grains but may have a contribution from colder dust in the background molecular cloud. The emission from FUV-irradiated warm dust is more easily detected at shorter FIR wavelengths. Indeed, the shell morphology in the PAH $8 \mu \mathrm{m}$ and PACS $70 \mu \mathrm{m}$ emission is very similar and nicely delineates the expanding shell. However, the bubble morphology is less apparent at longer submm wavelengths. Hence, to create an approximate map of $G_{0}$ along the line of sight toward the shell, shown in Fig. 8, we used:

$\log _{10} G_{0}=(0.975 \pm 0.02) \log _{10} I_{70}-(0.668 \pm 0.007)$,

where $I_{70}$ is the $70 \mu \mathrm{m}$ dust surface brightness in $\mathrm{MJy} \mathrm{sr}^{-1}$. We obtained this scaling after determining $G_{0}$ from SED fits toward the irradiated surface of OMC-1 (Goicoechea et al. 2015). Because photometric observations detect the dust continuum emission projected in the plane of the sky, it is not easy to resolve the dust temperature (or $G_{0}$ ) gradient along each line of sight. Hence, the $G_{0}$ contours shown in Fig. 8 should be understood as the maximum FUV flux that can impinge a globule located in a given position sightline. The minimum value $G_{0} \simeq 40$ (or $T_{\mathrm{d}, \mathrm{PDR}} \simeq 25 \mathrm{~K}$ ) is representative of the most distant shell edges far from the Trapezium. This $G_{0}$ value likely represents the local FUV flux around the negative- $v_{\text {LSR }}$ globules.

\subsection{Depth-dependent globule photochemical models}

In this section we go beyond the single-slab analysis and model the possible abundance and temperature gradients across a FUVirradiated globule. We use the Meudon PDR code (Le Petit et al. 2006) to model the penetration of FUV radiation (Goicoechea \& Le Bourlot 2007), thermal balance (Bron et al. 2014), steadystate gas chemistry, and NLTE [C II] $158 \mu \mathrm{m},{ }^{12} \mathrm{CO}$, and ${ }^{13} \mathrm{CO}$ excitation and radiative transfer (Gonzalez Garcia et al. 2008). We adopted an extinction-to-color-index ratio, $R_{\mathrm{V}}=A_{\mathrm{V}} / E_{\mathrm{B}-\mathrm{V}}$, of 5.5 (consistent with the flatter extinction curve observed in the 
Table 3. Parameters used in the PDR models of the negative- $v_{\text {LSR }}$ globules and comparison with observed intensities.

\begin{tabular}{|c|c|c|}
\hline Model parameter & Value & Note \\
\hline Local $G_{0}$ & 40 Habing & Best model \\
\hline Total depth $A_{\mathrm{V}}$ & $1.8 \mathrm{mag}$ & \\
\hline Gas density $n_{\mathrm{H}}$ & $2 \times 10^{4} \mathrm{~cm}^{-3}$ & \\
\hline Cosmic ray $\zeta_{\mathrm{CR}}$ & $10^{-16} \mathrm{H}_{2} \mathrm{~s}^{-1}$ & (a) \\
\hline$R_{\mathrm{V}}=A_{\mathrm{V}} / E_{\mathrm{B}-\mathrm{V}}$ & 5.5 & Orion $^{(b)}$ \\
\hline$N_{\mathrm{H}} / A_{\mathrm{V}}$ & $2.86 \times 10^{21} \mathrm{~cm}^{-2} \mathrm{mag}^{-1}$ & Orion $^{(b)}$ \\
\hline$M_{\text {gas }} / M_{\text {dust }}$ & 100 & Local ISM \\
\hline Abundance $\mathrm{O} / \mathrm{H}$ & $3.2 \times 10^{-4}$ & \\
\hline Abundance ${ }^{12} \mathrm{C} / \mathrm{H}$ & $1.5 \times 10^{-4}$ & Orion $^{(c)}$ \\
\hline Abundance N/H & $7.5 \times 10^{-5}$ & \\
\hline Abundance S / H & $1.5 \times 10^{-5}$ & \\
\hline${ }^{12} \mathrm{C} /{ }^{13} \mathrm{C}$ & 67 & Orion $^{(d)}$ \\
\hline Species & Predicted $N\left(\mathrm{~cm}^{-2}\right)$ & \\
\hline $\mathrm{C}^{+}$ & $3.5 \times 10^{17}$ & Best model \\
\hline${ }^{12} \mathrm{CO}$ & $5.2 \times 10^{15}$ & Best model \\
\hline${ }^{13} \mathrm{CO}$ & $1.8 \times 10^{14}$ & Best model \\
\hline Line & $\begin{array}{l}\text { Predicted } W \\
\left(\mathrm{~K} \mathrm{~km} \mathrm{~s}^{-1}\right)\end{array}$ & $\begin{array}{l}\text { Observed } \\
\left(\mathrm{K} \mathrm{km} \mathrm{s}^{-1}\right)\end{array}$ \\
\hline$[\mathrm{C}$ II $] 158 \mu \mathrm{m}$ & 32.3 & $29.3^{(\dagger)} \pm 6.0^{(\ddagger)}$ \\
\hline${ }^{12} \mathrm{CO}(2-1)$ & 10.8 & $9.5^{(\dagger)} \pm 4.3^{(\ddagger)}$ \\
\hline${ }^{13} \mathrm{CO}(2-1)$ & 0.4 & $0.7^{(\dagger)} \pm 0.3^{(\ddagger)}$ \\
\hline
\end{tabular}

Notes. ${ }^{(\dagger)}$ Mean line intensities toward globules \#1, \#2, and \#3. (‡) Standard deviation.

References. ${ }^{(a)}$ From Indriolo et al. (2015). ${ }^{(b)}$ From Lee (1968) and Cardelli et al. (1989). ${ }^{(c)}$ From Sofia et al. (2004). ${ }^{(d)}$ From Langer et al. (1984).

material toward the Trapezium stars in Orion, Lee 1968; Cardelli et al. 1989), and a $A_{\mathrm{V}} / N_{\mathrm{H}}$ ratio of $3.5 \times 10^{-22} \mathrm{mag} \mathrm{\textrm {cm } ^ { 2 }}$ appropriate for the material in Orion's Veil (e.g., Abel et al. 2016, 2019). The adopted model parameters and elemental abundances are tabulated in Table 3.

The chemical network includes specific ${ }^{13} \mathrm{C}$ isotopic fractionation reactions (Langer et al. 1984) meaning that the ${ }^{13} \mathrm{CO}$ predictions are accurate. In FUV-irradiated gas reaction ${ }^{13} \mathrm{C}^{+}+\mathrm{CO} \rightleftarrows \mathrm{C}^{+}+{ }^{13} \mathrm{CO}+\Delta E$ (1) (where $\Delta E=35 \mathrm{~K}$ is the zero-point energy difference between the species on either side of the reaction, Watson et al. 1976) transfers ${ }^{13} \mathrm{C}^{+}$ions and makes ${ }^{13} \mathrm{CO}$ more abundant than the ${ }^{12} \mathrm{CO} / R_{12 \mathrm{C} 13}$ ratio if $T_{\mathrm{k}}$ reaches $\sim 50 \mathrm{~K}$ and below (e.g., Röllig \& Ossenkopf 2013).

In our models, one side of the globule is illuminated by $G_{0} \geq 40$. The other side of the globule is illuminated by $G_{0}=1.7$. Starting around the extinction depths and column densities estimated from the single-slab analysis, we varied the local flux of FUV photons impinging the globule, the gas density $n_{\mathrm{H}}$ (first assumed to be constant), and $A_{\mathrm{V}}$ (the depth into the $\mathrm{CO}$ globule in magnitudes of visual extinction), and tried to fit the observed [C II] $158 \mu \mathrm{m},{ }^{12} \mathrm{CO}(2-1)$, and ${ }^{13} \mathrm{CO}$ (2-1) line intensities. Best model parameters and line emission predictions for the negative- $v_{\mathrm{LSR}}$ globules are shown in Table 3 .

The best-fit parameters to the observed line emission from the negative- $v_{\text {LSR }}$ globules (\#1, \#2, and \#3) are: $G_{0} \approx 40$, $n_{\mathrm{H}} \simeq 2 \times 10^{4} \mathrm{~cm}^{-3}$, and $A_{\mathrm{V}} \simeq 1.8 \mathrm{mag}$, giving a spatial size of
$2.5 \times 10^{17} \mathrm{~cm}=8300 \mathrm{AU} \simeq 2 R_{\mathrm{g}}$. This length scale approximately agrees with the observed radii of these globules. We note that an isobaric model (constant thermal pressure and varying density) with $P_{\text {th }} / k \simeq 10^{6} \mathrm{~cm}^{-3} \mathrm{~K}$ and $G_{0} \simeq 50$ predicts roughly the same intensities as the above constant density model. This thermal pressure is a good compromise between the values of $P_{\mathrm{th}} / k\left(\mathrm{C}^{+}\right)$ and $P_{\mathrm{th}, \mathrm{g}} / k$ (the internal globule pressure) inferred in the singleslab approximation (Table 2). We note that $G_{0}$ in these models is the local FUV flux around the globule needed to reproduce the observed line intensities, whereas the $G_{0}$ map in Fig. 8 shows the integrated FUV flux along the line of sight. The lower local $G_{0}$ values are consistent with the fact that these globules are embedded in neutral gas and dust that attenuates the FUV photon flux irradiating the shell.

The abundance, gas temperature, column density, and line emissivity profiles predicted by the constant density model are shown in Fig. 9 (left). Figure A.11 specifically compares the predicted and observed line intensities for the negative- $v_{\mathrm{LSR}}$ globules individually. These, and also the small globule \#6 (which is close in LSR velocity and shows similar emission properties), can be fitted within a factor three of the estimated local $G_{0}$ value (40), and within a factor three of the estimated gas density $\left(n_{\mathrm{H}}=2 \times 10^{4} \mathrm{~cm}^{-3}\right)$. This density is up to $\sim 10-40$ times higher than the density in the most common portions of the expanding shell, which do not show CO emission (see Abel et al. 2019, for their component III(B) of the Veil associated with the neutral shell).

As observed, our PDR models of the negative- $v_{\text {LSR }}$ globules predict that the $\mathrm{C}^{+} / \mathrm{CO}$ abundance ratio throughout the globule is above one, with a maximum abundance $x(\mathrm{CO})$ of several $10^{-6}$. Also, as observed, the model predicts an enhancement of the ${ }^{13} \mathrm{CO}$ column density produced by isotopic fractionation. Indeed, close to the $\mathrm{CO}$ abundance peak (at about $A_{\mathrm{V}}=1.5 \mathrm{mag}$ for this combination of $G_{0}$ and $n_{\mathrm{H}}$ values) the gas temperature drops to $\sim 50 \mathrm{~K}$ and reaction (1) becomes the dominant formation route for ${ }^{13} \mathrm{CO}$. This chemical effect favors the overproduction of ${ }^{13} \mathrm{CO}$ and explains the detected ${ }^{13} \mathrm{CO}(2-1)$ emission from the globules but no $\left[{ }^{13} \mathrm{C}\right.$ II $]$ emission (S. Kabanovic, priv. comm.).

A few of the brighter positive- $v_{\text {LSR }}$ globules show much brighter $8 \mu \mathrm{m}$, [C II] $158 \mu \mathrm{m}$, and ${ }^{12} \mathrm{CO}(2-1)$ emission levels (Table A.2), up to $\sim 100 \mathrm{~K} \mathrm{~km} \mathrm{~s}^{-1}$ (see globules \#5 and \#10 and their $8 \mu \mathrm{m}$ and [C II] $158 \mu \mathrm{m}$ bright rims). These are compatible with PDR models of higher FUV irradiation doses $\left(G_{0} \gtrsim 500\right.$, i.e., globules that are more exposed to the unattenuated radiation field from the Trapezium), higher gas densities $\left(n_{\mathrm{H}} \gtrsim 4 \times 10^{4} \mathrm{~cm}^{-3}\right)$, and higher extinction depths $\left(A_{\mathrm{V}} \gtrsim 5 \mathrm{mag}\right)$. These higher $n_{\mathrm{H}}$ and $A_{\mathrm{V}}$ values are consistent with our detection of $\mathrm{C}^{18} \mathrm{O}(2-1)$ line emission only toward globules \#5 and \#10.

Finally, the right panels in Fig. 9 are PDR models for a representative position in the Veil shell where $\mathrm{CO}$ is not detected. We chose $G_{0}=200, n_{\mathrm{H}}=3 \times 10^{3} \mathrm{~cm}^{-3}$ and $A_{\mathrm{V}}=1.8 \mathrm{mag}$ (e.g., Abel et al. 2016, 2019). The predicted $\mathrm{C}^{+}$column density $\left(N\left(\mathrm{C}^{+}\right) \simeq 10^{18} \mathrm{~cm}^{-2}\right)$ and [C II] $158 \mu \mathrm{m}$ line intensity $\left(\sim 50 \mathrm{~K} \mathrm{~km} \mathrm{~s}^{-1}\right)$ are in line with the values typically observed across the shell (Pabst et al. 2019, 2020). However, the predicted $\mathrm{CO}$ column density is very low, $N(\mathrm{CO}) \simeq 4 \times 10^{13} \mathrm{~cm}^{-2}$ (most carbon is in the form of $\mathrm{C}^{+}$), and the expected ${ }^{12} \mathrm{CO}(2-1)$ line intensity $\left(\sim 0.08 \mathrm{~K} \mathrm{~km} \mathrm{~s}^{-1}\right)$ is too faint to be detected. We conclude that owing to the strong stellar FUV irradiation conditions, most of the translucent gas in the shell will typically have exceedingly low CO column densities. 

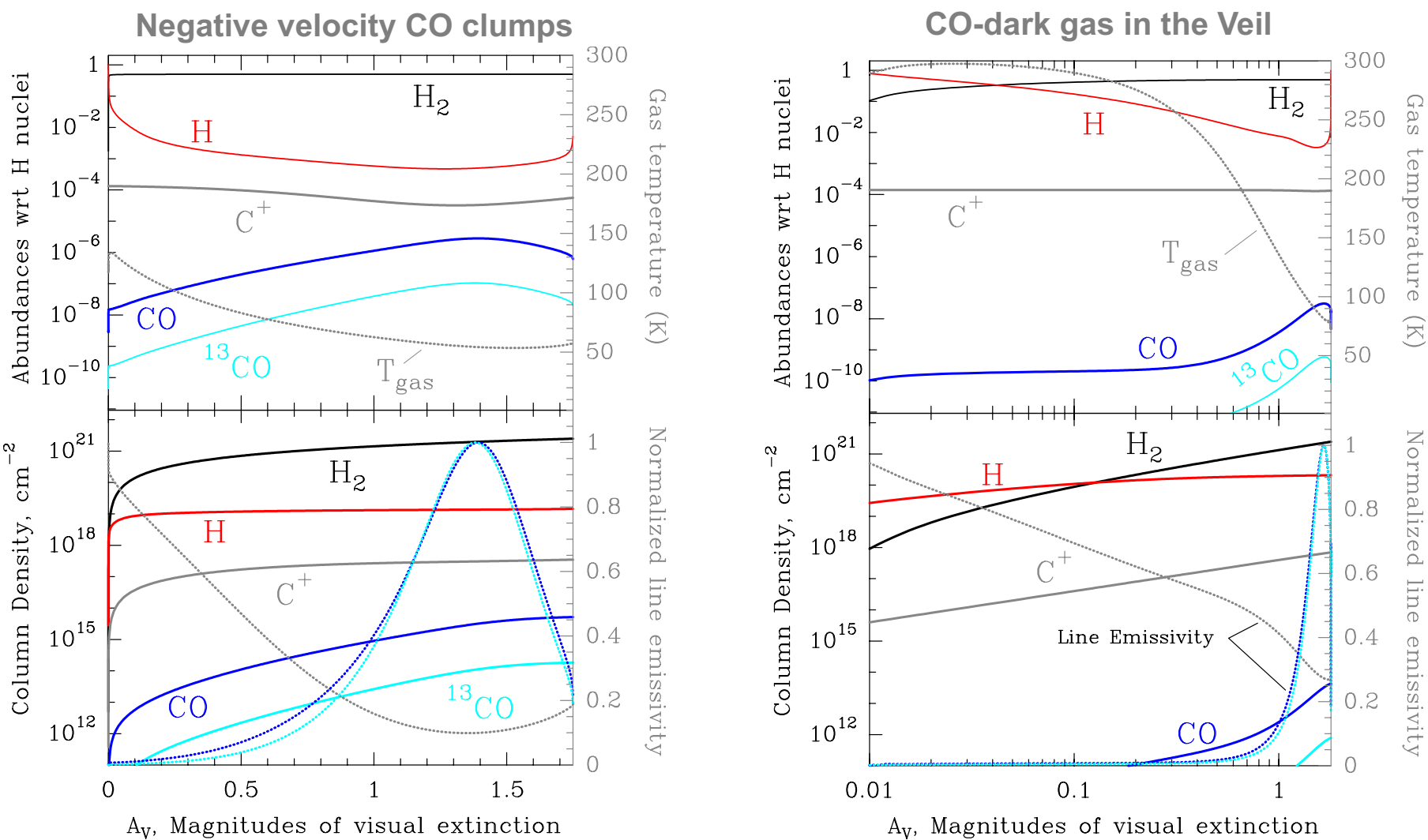

Fig. 9. Profiles of predicted abundance, gas temperature, column density, and line emissivity (dotted curve) as a function of globule depth. Left panel: model that reproduces the observed [C II] $158 \mu \mathrm{m},{ }^{12} \mathrm{CO}$ and ${ }^{13} \mathrm{CO}(2-1)$ line intensities toward the negative- $v_{\mathrm{LSR}} \mathrm{CO}$ globules (\#1, \#2, and \#3). The FUV field is $G_{0}=40$ and the gas density is $n_{\mathrm{H}}=2 \times 10^{4} \mathrm{~cm}^{-3}$. Right panel: model appropriate for a representative position of the shell where $\mathrm{CO}$ is not detected, with $n_{\mathrm{H}}=3 \times 10^{3} \mathrm{~cm}^{-3}, G_{0}=200$, and $A_{\mathrm{V}}=1.8$ (from Abel et al. 2019, and references therein).

\section{Discussion}

\subsection{Fraction of the shell mass traced by $C O$ globules}

In Pabst et al. (2019, 2020), we estimated the total mass of the expanding half-shell 1500-2600 $M_{\odot}$ (from the FIR dust opacity and from the [C II] $158 \mu \mathrm{m}$ emission itself). The region studied in the current work is about one-third of the shell and we determine that the shell mass in this area of interest is $\sim 400$ $700 M_{\odot}$. This is the mass enclosed inside the $T_{\mathrm{d}}>24 \mathrm{~K}$ contour shown in Fig. 10 (right panel). Here, $T_{\mathrm{d}}$ is an effective temperature obtained from a modified black body fit to the 70,100 , $160,250,350$, and $500 \mu \mathrm{m}$ photometric emission measured by Herschel (André et al. 2010). The morphology of the area with $T_{\mathrm{d}}>24 \mathrm{~K}$ resembles that revealed by the [C II] $158 \mu \mathrm{m}$, PACS 70, and PAH $8 \mu \mathrm{m}$ images that delineates the expanding shell. All $\mathrm{CO}$ globules lie inside the $T_{\mathrm{d}}>24 \mathrm{~K}$ area. We compute the mass of the $\mathrm{CO}$ globules by considering their observed sizes (as seen in ${ }^{12} \mathrm{CO}$ ) and their column densities determined from the singleslab analysis. We derive a total mass that ranges from 4 to $14 M_{\odot}$ adding their masses. Therefore, they only account for $<3 \%$ of the shell mass.

\subsection{Magnetic support and external pressure confinement}

The $\mathrm{CO}$ globules have the following median properties: radius $R_{\mathrm{g}} \simeq 7100 \mathrm{AU}$, gas density $n_{\mathrm{H}}=3.6 \times 10^{4} \mathrm{~cm}^{-3}$, and mass $M_{\mathrm{g}}=0.3 M_{\odot}$. The observed ${ }^{13} \mathrm{CO}$ line profiles (Fig. 5 and Table A.2) imply supersonic nonthermal motions inside them, with a median turbulent velocity dispersion of $\sigma_{\text {turb }}\left({ }^{13} \mathrm{CO}\right)=$ $0.5 \mathrm{~km} \mathrm{~s}^{-1}$. Turbulent pressure dominates thermal pressure in the globules (see Table 2 for each globule individually). The [C II] $158 \mu \mathrm{m}$ line profiles are systematically broader, indicating more extended gas flows, as well as higher turbulent velocity dispersions in their envelopes and in the interglobule medium, with a median of $\sigma_{\text {turb }}\left(\mathrm{C}^{+}\right)=1.3 \mathrm{~km} \mathrm{~s}^{-1}$. This value implies high turbulent pressures of $P_{\text {turb }}\left(\mathrm{C}^{+}\right)=\rho \sigma_{\text {turb }}^{2}$ (with $\left.\rho=\mu m_{\mathrm{H}} n_{\mathrm{H}}\right)$ around the $\mathrm{CO}$ globules. Their external pressure (thermal plus turbulent) is $P_{\text {ext }} / k \gtrsim 10^{7} \mathrm{~cm}^{-3} \mathrm{~K}$ (median), higher than their internal pressures $\left(P_{\mathrm{g}} / k\right.$ of a few $\left.10^{6} \mathrm{~cm}^{-3} \mathrm{~K}\right)$.

Velocity-resolved HI Zeeman observations toward thin, lowdensity $\left(n_{\mathrm{H}} \gtrsim 10^{3} \mathrm{~cm}^{-3}\right)$ portions of the Veil have suggested typical (line-of-sight) magnetic field strengths of $B_{\mathrm{los}}=50-75 \mu \mathrm{G}$, reaching $B_{\text {los }}=100 \mu \mathrm{G}$ or more toward several positions (Troland et al. 2016). This latter value implies magnetic pressures of $P_{\mathrm{B}} / k=B_{\text {tot }}^{2} / 8 \pi k \geq 8 \times 10^{6} \mathrm{~K} \mathrm{~cm}^{-3}$ if $B_{\text {los }}^{2}=B_{\text {tot }}^{2} / 3$ (Crutcher 1999; Abel et al. 2016). Hence, the Veil seems to be a very magnetized medium, with the energy in $B_{\text {tot }}$ being similar to or more than that in gas motions or gravity. In general, $B_{\text {tot }}$ increases with density at ionized and molecular gas boundaries $\left(B_{\mathrm{tot}} \propto n_{\mathrm{H}}^{0.5-1}\right.$, e.g., Planck Collaboration Int. XXXIV 2016). Hence, we can expect stronger fields threading the denser CO globules. As an example, the derived intensity of the (plane-of-the-sky) magnetic field in the Orion Bar PDR is $\sim 300 \mu \mathrm{G}$ (derived from the polarized FIR dust emission; Chuss et al. 2019). In the swept-up material that confines the bubble, compression associated with the expansion of the shell itself will further increase the magnetic field as, given the high degree of ionization in PDR gas, $n\left(e^{-}\right) \approx n\left(\mathrm{C}^{+}\right)$, the field lines will be "frozen-in". Overall, we conclude that plausible magnetic fields $\left(B_{\text {los }} \approx 100-200 \mu \mathrm{G}\right.$ ) would be sufficient to magnetically support the $\mathrm{CO}$ globules. We note that simulations 

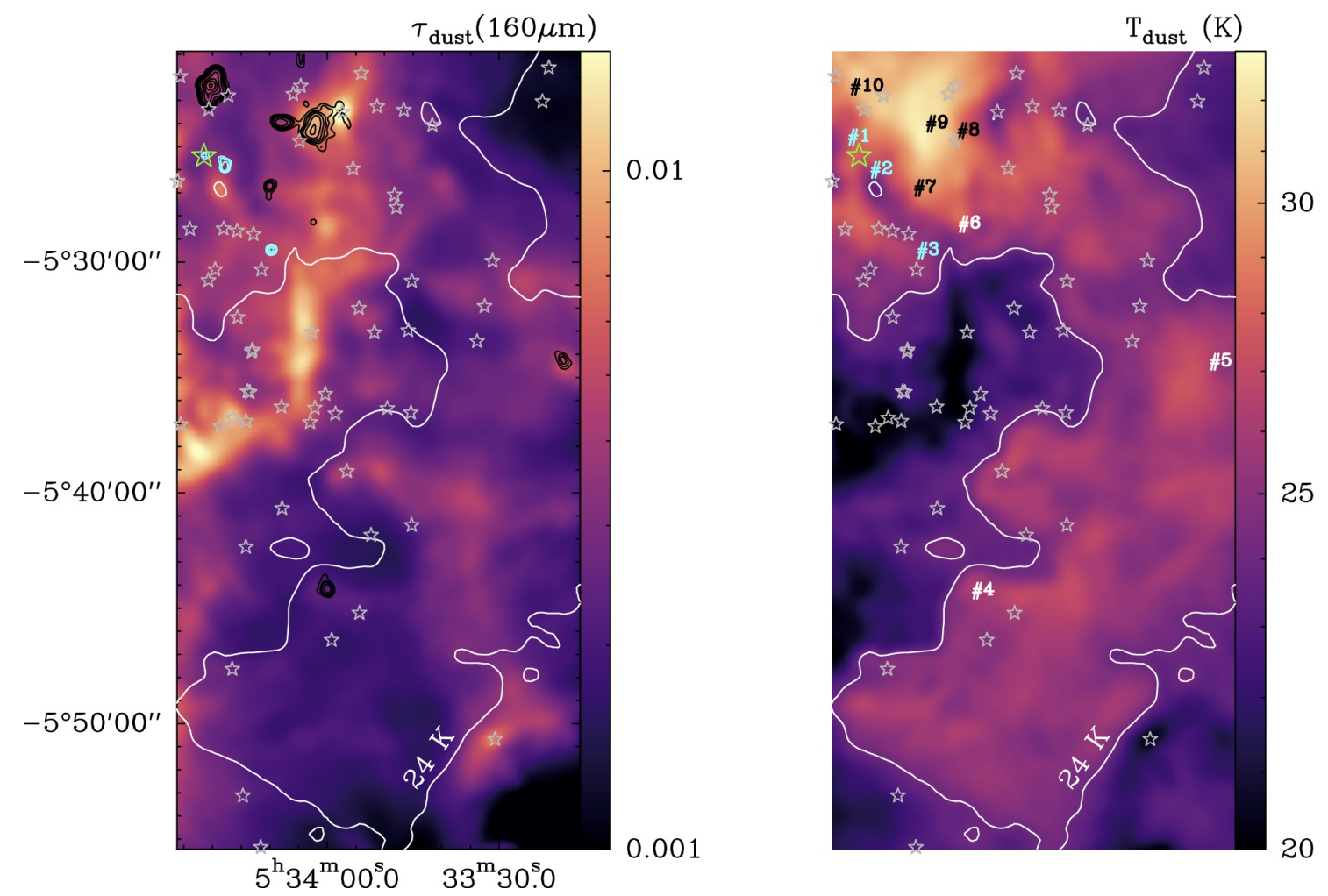

Fig. 10. Dust opacity at $160 \mu \mathrm{m}$ (left panel) and the dust temperature (right) computed by fitting modified blackbodies to the continuum emission measured by Herschel (Pabst et al. 2019). The white contour at $T_{\mathrm{d}}=24 \mathrm{~K}$ roughly encloses the expanding shell as observed in [C II] $158 \mu \mathrm{m}$ and $\mathrm{PAH}$ emission. The lower- $T_{\mathrm{d}}$ regions outside this area mostly arise from the background molecular cloud and not from the shell. Left panel: ${ }^{12} \mathrm{CO}$ (2-1) emission from the globules in cyan (black) contours for the negative (positive) LSR velocities. Right panel: their labels. In both maps, the gray stars show the position (in the plane of the sky) of YSOs previously detected from infrared observations (Megeath et al. 2012, 2016). Only globule \#1 matches the position of a YSO (object \#1728) shown with a green star.

do predict that FUV-irradiated cometary globules can be magnetically supported (e.g., Lefloch \& Lazareff 1994; Henney et al. 2009; Mackey \& Lim 2011).

The expanding shell is certainly massive, $1500-2600 M_{\odot}$ (Pabst et al. 2020), comparable to the current mass of the molecular core behind (OMC-1; e.g., Genzel \& Stutzki 1989; Bally et al. 1987). The shell material has been swept up by the disruptive effects of $\theta^{1}$ Ori $\mathrm{C}$ winds and UV radiation. However, in the surveyed area, the mass of $\mathrm{CO}$ globules only represents $<3 \%$ of the total shell mass. Assuming $G_{0}>40$, the timescale for photodissociation of $\mathrm{CO}$ in the shell is considerably shorter than its expansion timescale ( $\tau_{\exp } \simeq 200000 \mathrm{yr}$; Pabst et al. 2019). This is consistent with the lack of widespread and extended $\mathrm{CO}$ emission in the Veil and leaves the origin and fate of the detected molecular globules as an open question. We discuss this issue, at least qualitatively, in the following section.

\subsection{Origin and evolution of the CO globules in the Veil}

Globules may form in-situ by hydrodynamic instabilities (see e.g., Schneps et al. 1980; Sharp 1984; Murray et al. 1993; Nakamura et al. 2006) at the interface between the (shell's) neutral gas that rests on a light and rarefied (about $1 \mathrm{~cm}^{-3}$ ) hot plasma (Güdel et al. 2008). This interface is Rayleigh-Taylor unstable (Spitzer 1954) and could form "trunks" or "fingers" of size $R$ on timescales of $\tau_{\mathrm{RT}} \approx R / c_{\mathrm{s}} \simeq 10^{5}(R / 0.1 \mathrm{pc}$ ) $\mathrm{yr}$ (where $c_{\mathrm{S}}$ is the speed of sound in the shell: $1 \mathrm{~km} \mathrm{~s}^{-1}$ at $\left.100 \mathrm{~K}\right)$. The observed sizes of the CO globules, about $10 \%$ of the total shell thickness, indeed imply that they could form during the expansion of the shell into an environment of lower density
( $\tau_{\mathrm{RT}}<\tau_{\exp }$, see e.g., Schneps et al. 1980). Kelvin-Helmholtz instabilities could also develop at the side of the fingers over similar timescales (Sharp 1984; Murray et al. 1993; Nakamura et al. 2006; Berné \& Matsumoto 2012). Indeed, wave-like emission structures (typical of these instabilities) with spatial wavelengths ranging from 0.1 to $0.01 \mathrm{pc}$ have been observed at several ionized-molecular gas interfaces in OMC-1. In particular, Berné et al. (2010) and Berné \& Matsumoto (2012) analyzed the "KH ripples", with \#7 being the head of this structure (see Fig. 6 for a detailed view). Much higher resolution ALMA images of the Orion Bar PDR suggest smaller scale density undulations separated by $\sim 0.01 \mathrm{pc}$ at the FUV-irradiated boundary between OMC-1 and the Huygens H II region (Goicoechea et al. 2016).

Alternatively, the $\mathrm{CO}$ globules could have been pre-existing molecular structures (e.g., like in Reipurth 1983), which are denser than the shell, and are perhaps generated by the turbulent velocity field in the OMC (e.g., Hartmann \& Burkert 2007; Hacar et al. 2017). These globules are compressed and swept along by a passing shock accompanying the expanding shell (e.g., Pikel'Ner \& Sorochenko 1974). If the initial gas density contrast between globules and shell has a value of about 10, the shock wave penetrating the $\mathrm{CO}$ globules will be slower by a factor of approximately three. This gives a shock velocity of $v_{\text {shock }}=4 \mathrm{~km} \mathrm{~s}^{-1}$, fast enough to traverse the globule in $\tau_{\text {shock }} \approx R_{\mathrm{g}} / v_{\text {shock }} \simeq 10000 \mathrm{yr}$ and trigger compression. Gas cooling through CO lines will decrease the post-shock gas temperature and further enhance gas compression and density. This could result in free-fall times, $t_{\mathrm{ff}} \approx(G \rho)^{-1 / 2}$, comparable to the shell expansion timescale $\left(\tau_{\exp }\right)$ if globules are compressed to 
densities $>2 \times 10^{4} \mathrm{~cm}^{-3}$ (comparable with our estimated values). However, given the physical conditions in the Veil shell: enhanced turbulence, FUV-heating, and strong magnetic field, it may be unlikely that gravity can beat the pressure support of the globule (see further discussion in the following section).

The detected $\mathrm{CO}$ globules are surrounded by larger structures of predominantly neutral gas and dust, emitting thermal continuum at $70 \mu \mathrm{m}$, PAHs, and [C II] $158 \mu \mathrm{m}$ (that matches the velocity of the $\mathrm{CO}$ emission; see Fig. A.1). This material likely shields the globules from extreme UV (EUV; $E>13.6 \mathrm{eV}$ ) ionizing radiation and reduces the flux of dissociating FUV photons reaching their interiors. This scenario is consistent with the observed low CO excitation temperatures $(\sim 10 \mathrm{~K})$ and moderate local $G_{0}$ values around the negative- $v_{\mathrm{LSR}}$ globules (and \#6). It is also consistent with their apparent spherical morphology (globules \#1, \#4 and \#6). This is indicative of little active interaction with ionizing EUV photons that would have carved them into "tear drops" and less spherical shapes (e.g., De Marco et al. 2006). This would also explain why these globules survive (longer) in this harsh environment. Alternatively, some of the more roundish globules might have already lost their lower density molecular tails (e.g., Gahm et al. 2013). We find this scenario less likely for globules currently embedded in the shell, but this may be the case for globule \#3 which looks more isolated in its position-velocity diagram (Fig A.4) and is perhaps located outside the shell. However, the shell geometry is more complicated than the simple modeled arcs shown in the diagrams, and therefore this argument is not conclusive. In addition, and even if the detected globules in Orion are all mostly illuminated laterally, projection effects may influence their apparent morphologies (that depend on the aspect angle). In extreme cases, an elongated globule with head or tail may look spherical.

Eventually, the ionization front associated with the expanding $\mathrm{H}$ II region inside the expanding bubble will encounter these globules, further sculpting, photoevaporating, and compressing them (see simulations in Henney et al. 2009). This radiative interaction will lead to a significant erosion of the globules (e.g., Bertoldi \& McKee 1990; Lefloch \& Lazareff 1994). The smallest and roundish globules may develop "cometary" or "tear drop" shapes. Still, their morphological evolution depends on the strength and orientation of the magnetic field threading them. For strong fields, simulations predict that photoevaporating globules can acquire flattened shapes (Henney et al. 2009). Only globule \#9 is suggestive of this geometry.

On the other hand, the larger and more elongated $\mathrm{CO}$ structures at positive- $v_{\mathrm{LSR}}$ (globules $\# 5$, \#8, and \#10) represent the first stages in the formation of (Orion-equivalent) miniature "Pillars of Creation" (e.g., Pound 1998; Tremblin et al. 2012). Globules \#5 and \#8 are located at the limb-brightened edge of the shell (and their CO emission is connected to that of the background molecular cloud) whereas \#10 is not at the limb and it is more isolated in velocity with respect to the OMC. These structures, and also the "KH ripples" (Berné et al. 2010), display bright $8 \mu \mathrm{m}$ and [C II] $158 \mu \mathrm{m}$ emission rims pointing toward the Trapezium (see Figs. 6 and A.1). They are also characterized by higher CO excitation temperatures $(\sim 40-60 \mathrm{~K})$ and higher local $G_{0}$ values (up to hundreds). Hence, they are in a more active phase of radiative shaping and new small globules may detach from them.

\subsection{Low-mass star-formation in the Veil?}

An intriguing question is whether this kind of small globule can form new stars of very low mass. For several of the detected globules in Orion, their external pressure seems sufficient to overcome the stability limit for Bonnor-Ebert spheres (see Table 2) and so they do not seem to be in hydrostatic equilibrium but instead to be dynamically evolving (e.g., Galli et al. 2002). However, the estimated globule masses are smaller than the Jeans mass needed for gravity to dominate and trigger collapse (Sect. 4.2). Indeed, the inferred large globule virial parameters, $\alpha=5 \sigma^{2} R_{\mathrm{g}} / G M_{\mathrm{g}} \gg 1$, the derived scaling of $\alpha$ with their mass, $\alpha \propto M_{\mathrm{g}}^{-0.6 \pm 0.1}$, and the likely fact that $P_{\mathrm{ext}} \approx P_{\mathrm{B}, \mathrm{g}}$ all agree with theoretical expectations of pressure-confined, gravitationally unbound globules (e.g., Bertoldi \& McKee 1992; Lada et al. 2008).

Interestingly, while the dense prestellar cores in Orion's integral-shape filament are confined by pressure due to the weight of the molecular cloud and the filament (Kirk et al. 2017), the CO globules in the Veil are mainly confined by the turbulent pressure of the wind-driven shell. This points to transient molecular globules that would ultimately be photoevaporated (e.g., Oort \& Spitzer 1955; Gorti \& Hollenbach 2002) or expand and disperse as the pressure inside the expanding shell decreases. In this likely scenario, most $\mathrm{CO}$ globules, and the bulk material in the shell, will not form new stars. Given the large masses swept up in the shell, this will limit the global star-formation rate in Orion.

The exception that confirms the rule is globule \#1 (shown in detail in Fig. 6). Its position coincides with a known young stellar object that was classified as a pre-main sequence star with a disk and was detected in previous infrared imaging surveys of Orion (YSO \#1728 of Megeath et al. 2012). This match suggests that some low-mass protostars do exist inside the shell. In these cases, their molecular cocoons were probably dense and massive enough before being engulfed by the expanding shell.

The more isolated and smaller CO globules detected in the shell are part of an interesting class of tiny molecular clouds $\left(\sim 0.1 M_{\odot} \simeq 100 M_{\text {Jup }}\right)$ that are different from the bigger and more massive "Bok globules". The latter ones usually form one or a few low-mass stars but they are mostly unrelated to $\mathrm{H}$ II regions (Bok \& Reilly 1947; Reipurth 1983; Nelson \& Langer 1999; Launhardt et al. 2010). Previous studies of small globules around the H II region in the Rosette Nebula have suggested that they could be a source of brown dwarfs and free-floating planetarymass objects (e.g., Gahm et al. 2007, 2013). However, our virial and Jeans mass analysis does not support this scenario for the small globules in Orion. Nevertheless, it would be interesting to carry out follow-up deep observations of these objects in higher critical-density molecular tracers able to reveal the presence of denser and more massive gas cores in their interiors.

\section{Summary and conclusions}

We expand previous maps of Orion A taken with the IRAM $30 \mathrm{~m}$ telescope in the ${ }^{12} \mathrm{CO},{ }^{13} \mathrm{CO}$, and $\mathrm{C}^{18} \mathrm{O}(\mathrm{J}=2-1)$ lines at $11^{\prime \prime}$ resolution $(\simeq 4500 \mathrm{AU})$. We investigate a $2 \mathrm{pc} \times 4 \mathrm{pc}\left(\sim 600 \operatorname{arcmin}^{2}\right)$ region of the neutral shell that confines the wind-driven Veil bubble around the Trapezium cluster. This massive shell, which is made of swept up material from the natal Orion molecular cloud, is very bright in [C II] $158 \mu \mathrm{m}$, PAHs, and $70 \mu \mathrm{m}$ dust emission (Pabst et al. 2019). Owing to intense UV radiation from $\theta^{1}$ Ori $C$, the most massive O-type star in the cluster, the expected column densities of molecular gas in this extended but translucent foreground material were very low. We summarize the primary results of this work as follows:

- We find that widespread and extended $\mathrm{CO}$ emission is largely absent from the Veil. This implies that most of the neutral 
material that surrounds the Orion cluster is "CO-dark" but not necessarily $100 \%$ atomic. In particular, we present the detection of CO globules (some of them at negative $v_{\mathrm{LSR}}$ ) embedded in the expanding shell that encloses the bubble.

- The CO globules are small $\left(R_{\mathrm{g}} \simeq 7100 \mathrm{AU}\right)$, moderately dense $\left(n_{\mathrm{H}}=3.6 \times 10^{4} \mathrm{~cm}^{-3}\right)$, and have low masses: $M_{\mathrm{g}}=0.3 M_{\odot}$ (median values of the sample). The observed [C II] $158 \mu \mathrm{m}$, ${ }^{12} \mathrm{CO}$, and ${ }^{13} \mathrm{CO}(2-1)$ emission from the negative- $v_{\mathrm{LSR}}$ globules (\#1, \#2, and \#3) and also from \#6, those closer to us, are reproduced by depth-dependent PDR models with $n_{\mathrm{H}} \approx 2 \times 10^{4} \mathrm{~cm}^{-3}$ and $G_{0} \approx 40$ (both quantities within a factor 3 ). These globules show roundish morphologies. In the other extreme of the sample, structures \#5 and \#10 (at positive $v_{\mathrm{LSR}}$ ) are bigger and more elongated. Fitting their emission lines requires stronger FUV photon fluxes $\left(G_{0} \gtrsim 500\right)$, higher densities $\left(n_{\mathrm{H}} \gtrsim 4 \times 10^{4} \mathrm{~cm}^{-3}\right)$, and higher extinction depths $\left(A_{\mathrm{V}} \gtrsim 5 \mathrm{mag}\right)$. These values are consistent with the detection of $\mathrm{C}^{18} \mathrm{O}(2-1)$ emission from these two molecular gas structures.

The inferred nonthermal velocity dispersions $\left(\sigma_{\text {turb }}\left(\mathrm{C}^{+}\right) \simeq 1.3 \mathrm{~km} \mathrm{~s}^{-1}\right.$ and $\left.\sigma_{\text {turb }}\left({ }^{13} \mathrm{CO}\right) \simeq 0.5 \mathrm{~km} \mathrm{~s}^{-1}\right)$ indicate that the gas in the shell and in the envelopes around the globules is more turbulent and warmer $\left(T_{\mathrm{k}} \approx 100 \mathrm{~K}\right)$ than in their molecular interiors $\left(T_{\mathrm{k}} \approx 10-50 \mathrm{~K}\right)$. These dispersions imply transonic to supersonic motions. The ratio of the nonthermal to thermal pressure is always greater than one (and up to $\sim 10$ ) both in the globule interiors and in their envelopes. We derive that the external pressure around the $\mathrm{CO}$ globules (turbulent + thermal) is high, $P_{\text {ext }} / k \gtrsim 10^{7} \mathrm{~K} \mathrm{~cm}^{-3}$ (median value).

- The magnetic field strength previously derived in lowdensity portions of the Veil and in the denser gas of the Orion Bar suggests that the $\mathrm{CO}$ globules in the shell can be threaded by fields of the order of $100-200 \mu \mathrm{G}$. These values would be sufficient to magnetically support them $\left(P_{\text {ext }} \simeq P_{\mathrm{B}, \mathrm{g}}\right)$. Together with the inferred large virial parameters, $\alpha \gg 1$, the gathered evidence suggests pressure-confined, gravitationally unbound globules.

- The CO globules are embedded in the predominantly neutral material of the shell. They are either transient objects formed by hydrodynamic instabilities or pre-existing over-dense structures of the original molecular cloud. They are being sculpted by the passing shock associated with the expanding shell and by UV radiation from $\theta^{1}$ Ori C. Several of them are isolated and intriguingly roundish. This suggests that they are not being actively photo-ionized. The more elongated and warmer globules (\#5, \#8, and \#10) likely represent the first stages in the formation of (Orion-equivalent) miniature Pillars of Creation.

- The estimated masses of all globules are lower than their Jeans masses, and thus do not imply they will easily form stars unless they accrete more mass. To break the rule, globule \#1 coincides with the position of a known YSO. This suggests that some low-mass protostars do exist inside the expanding shell. One possibility is that their molecular cores were massive enough and had collapsed before being caught by the shell.

The lack of extended CO emission from the swept-up shell that encloses the Veil bubble in Orion implies that winds and UV radiation from young massive stars expel, agitate, and photodissociate most of the disrupted molecular cloud gas. This material is blown to large distances, far from the main starforming cores. Large-scale turbulence and FUV heating across the shell hinders the formation of new stars. Given the large amount of disrupted material, these feedback processes must limit the star-formation rate in Orion. M42 is certainly impressive (mostly because it is so close to us) but it is interesting to see that most of the fireworks, energetics, and gas dynamics are mostly driven by the massive stellar system $\theta^{1}$ Ori $\mathrm{C}$.
Acknowledgements. We acknowledge helpful comments and suggestions from our referee. We warmly thank the operators, AoDs, and chefs at the IRAM $30 \mathrm{~m}$ telescope for their support while the $\mathrm{CO}$ observations were conducted. This work is also based on observations made with the NASA/DLR Stratospheric Observatory for Infrared Astronomy (SOFIA). SOFIA is jointly operated by the Universities Space Research Association, Inc. (USRA), under NASA contract NNA17BF53C, and the Deutsches SOFIA Institut (DSI) under DLR contract 50 OK 0901 to the University of Stuttgart. We acknowledge the work, during the $\mathrm{C}^{+}$upGREAT square degree survey of Orion, of the USRA and NASA staff of the Armstrong Flight Research Center in Palmdale, the Ames Research Center in Mountain View (California), and the Deutsches SOFIA Institut. We thank the Spanish MICIU for funding support under grant AYA2017-85111-P. Research on the ISM at Leiden Observatory is supported through a Spinoza award.

\section{References}

Abel, N. P., Ferland, G. J., O’Dell, C. R., Shaw, G., \& Troland, T. H. 2006, ApJ, 644, 344

Abel, N. P., Ferland, G. J., O’Dell, C. R., \& Troland, T. H. 2016, ApJ, 819, 136

Abel, N. P., Ferland, G. J., \& O'Dell, C. R. 2019, ApJ, 881, 130

André, P., Men'shchikov, A., Bontemps, S., et al. 2010, A\&A, 518, L102

Bally, J. 2008, Handbook of Star Forming Regions, ed. B. Reipurth ASP: California, 459

Bally, J., Langer, W. D., Stark, A. A., \& Wilson, R. W. 1987, ApJ, 312, L45

Berné, O., \& Matsumoto, Y. 2012, ApJ, 761, L4

Berné, O., Marcelino, N., \& Cernicharo, J. 2010, Nature, 466, 947

Berné, O., Marcelino, N., \& Cernicharo, J. 2014, ApJ, 795, 13

Bertoldi, F., \& McKee, C. F. 1990, ApJ, 354, 529

Bertoldi, F., \& McKee, C. F. 1992, ApJ, 395, 140

Bok, B. J., \& Reilly, E. F. 1947, ApJ, 105, 255

Bolatto, A. D., Wolfire, M., \& Leroy, A. K. 2013, ARA\&A, 51, 207

Bonnor, W. B. 1956, MNRAS, 116, 351

Boreiko, R. T., \& Betz, A. L. 1996, ApJ, 467, L113

Bron, E., Le Bourlot, J., \& Le Petit F. 2014, A\&A, 569, A100

Buckle, J. V., Davis, C. J., di Francesco, J., et al. 2012, MNRAS, 422, 521

Cardelli, J. A., Clayton, G. C., \& Mathis, J. S. 1989, ApJ, 345, 245

Carter, M., Lazareff, B., Maier, D., et al. 2012, A\&A, 538, A89

Cernicharo, J., \& Guelin, M. 1987, A\&A, 176, 299

Churchwell, E., Smith, L. F., Mathis, J., Mezger, P. G., \& Huchtmeier, W. 1978 A\&A, 70, 719

Churchwell, E., Povich, M. S., Allen, D., et al. 2006, ApJ, 649, 759

Chuss, D. T., Andersson, B. G., Bally, J., et al. 2019, ApJ, 872, 187

Crutcher, R. M. 1999, ApJ, 520, 706

Cuadrado, S., Salas, P., Goicoechea, J. R., et al. 2019, A\&A, 625, L3

Deharveng, L., Schuller, F., Anderson, L. D., et al. 2010, A\&A, 523, A6

De Marco, O., O’Dell, C. R., Gelfond, P., Rubin, R. H., \& Glover, S. C. O. 2006, AJ, 131, 2580

Dent, W. R. F., Hovey, G. J., Dewdney, P. E., et al. 2009, MNRAS, 395, 1805

Ebert, R. 1955, Z. Astrophys., 37, 217

Frerking, M. A., Langer, W. D., \& Wilson, R. W. 1982, ApJ, 262, 590

Gahm, G. F., Grenman, T., Fredriksson, S., \& Kristen, H. 2007, AJ, 133 , 1795

Gahm, G. F., Persson, C. M., Mäkelä, M. M., \& Haikala, L. K. 2013, A\&A, 555, A57

Galli, D., Walmsley, M., \& Gonçalves, J. 2002, A\&A, 394, 275

Genzel, R., \& Stutzki, J. 1989, ARA\&A, 27, 41

Goicoechea, J. R., \& Le Bourlot J. 2007, A\&A, 467, 1

Goicoechea, J. R., Pety, J., Cuadrado, S., et al. 2016, Nature, 537, 207

Goicoechea, J. R., Teyssier, D., Etxaluze, M., et al. 2015, ApJ, 812, 75

Goicoechea, J. R., Santa-Maria, M. G., Bron, E., et al. 2019, A\&A, 622, A91

Gonzalez-Alfonso, E., \& Cernicharo, J. 1994, ApJ, 430, L125

Gonzalez Garcia, M., Le Bourlot, J., Le Petit, F., \& Roueff, E. 2008, A\&A, 485, 127

Gorti, U., \& Hollenbach, D. 2002, ApJ, 573, 215

Gravity Collaboration (Karl M., et al.) 2018, A\&A, 620, A116

Grenier, I. A., Casandjian, J.-M., \& Terrier, R. 2005, Science, 307, 1292

Greve, A., Kramer, C., \& Wild, W. 1998, A\&AS, 133, 271

Güdel, M., Briggs, K. R., Montmerle, T., et al. 2008, Science, 319, 309

Habing, H. J. 1968, Bull. Astron. Inst. Netherlands, 19, 421

Hacar, A., Alves, J., Tafalla, M., \& Goicoechea, J. R. 2017, A\&A, 602, L2

Haid, S., Walch, S., Seifried, D., et al. 2018, MNRAS, 478, 4799

Hartmann, L., \& Burkert, A. 2007, ApJ, 654, 988 
Henney, W. J., Arthur, S. J., de Colle, F., \& Mellema, G. 2009, MNRAS, 398, 157

Herbig, G. H. 1974, PASP, 86, 604

Hollenbach, D. J., \& Tielens, A. G. G. M. 1997, ARA\&A, 35, 179

Hollenbach, D. J., Takahashi, T., \& Tielens, A. G. G. M. 1991, ApJ, 377, 192

Huggins, P. J., Bachiller, R., Cox, P., \& Forveille, T. 1992, ApJ, 401, L43

Huggins, P. J., Forveille, T., Bachiller, R., et al. 2002, ApJ, 573, L55

Indriolo, N., Neufeld, D. A., Gerin, M., et al. 2015, ApJ, 800, 40

Johnstone, D., \& Bally, J. 1999, ApJ, 510, L49

Kirk, H., Friesen, R. K., Pineda, J. E., et al. 2017, ApJ, 846, 144

Kong, S., Arce, H. G., Feddersen, J. R., et al. 2018, ApJS, 236, 25

Krumholz, M. R., Bate, M. R., Arce, H. G., et al. 2014, Protostars and Planets VI (Tucson: University of Arizona Press), 243

Lada, C. J., Muench, A. A., Rathborne, J., Alves, J. F., \& Lombardi, M. 2008, ApJ, 672, 410

Langer, W. D., \& Penzias, A. A. 1990, ApJ, 357, 477

Langer, W. D., Graedel, T. E., Frerking, M. A., \& Armentrout, P. B. 1984, ApJ, 277,581

Larson, R. B. 1978, MNRAS, 184, 69

Launhardt, R., Nutter, D., Ward-Thompson, D., et al. 2010, ApJS, 188, 139

Lee, T. A. 1968 , ApJ, 152, 913

Lefloch, B., \& Lazareff, B. 1994, A\&A, 289, 559

Le Petit, F., Nehmé, C., Le Bourlot, J., \& Roueff, E. 2006, ApJS, 164, 506

Mackey, J., \& Lim, A. J. 2011, MNRAS, 412, 2079

Madden, S. C., Poglitsch, A., Geis, N., Stacey, G. J., \& Townes, C. H. 1997, ApJ, 483, 200

Megeath, S. T., Gutermuth, R., Muzerolle, J., et al. 2012, AJ, 144, 192

Megeath, S. T., Gutermuth, R., Muzerolle, J., et al. 2016, AJ, 151, 5

Menten, K. M., Reid, M. J., Forbrich, J., \& Brunthaler, A. 2007, A\&A, 474, 515

Minkowski, R. 1949, PASP, 61, 151

Murray, S. D., White, S. D. M., Blondin, J. M., \& Lin, D. N. C. 1993, ApJ, 407, 588

Nakamura, F., McKee, C. F., Klein, R. I., \& Fisher, R. T. 2006, ApJS, 164, 477

Natta, A., Walmsley, C. M., \& Tielens, A. G. G. M. 1994, ApJ, 428, 209

Nelson, R. P., \& Langer, W. D. 1999, ApJ, 524, 923

O’Dell, C. R. 2001, ARA\&A, 39, 99

Oort, J. H. \& Spitzer, L., Jr. 1955, ApJ, 121, 6
Ossenkopf, V., Röllig, M., Neufeld, D. A., et al. 2013, A\&A, 550, A57 Pabst, C., Higgins, R., Goicoechea, J. R., et al. 2019, Nature, 565, 618 Pabst, C., Goicoechea, J. R., Teyssier, D., et al. 2020, A\&A, 639, A2 Penzias, A. A., \& Burrus, C. A. 1973, ARA\&A, 11, 51

Pety, J., Guzmán, V. V., Orkisz, J. H., et al. 2017, A\&A, 599, A98

Phillips, T. G., Huggins, P. J., Wannier, P. G., \& Scoville, N. Z. 1979, ApJ, 231, 720

Pikel'Ner, S. B., \& Sorochenko, R. L. 1974, Sov. Astron., 17, 443

Planck Collaboration XIX. 2011, A\&A, 536, A19

Planck Collaboration Int. XXXIV. 2016, A\&A, 586, A137

Pound, M. W. 1998, ApJ, 493, L113

Rahner, D., Pellegrini, E. W., Glover, S. C. O., \& Klessen, R. S. 2017, MNRAS, 470, 4453

Reipurth, B. 1983, A\&A, 117, 183

Rodríguez-Franco, A., Martin-Pintado, J., \& Fuente, A. 1998, A\&A, 329, 1097

Röllig, M., \& Ossenkopf, V. 2013, A\&A, 550, A56

Salas, P., Oonk, J. B. R., Emig, K. L., et al. 2019, A\&A, 626, A70

Schneps, M. H., Ho, P. T. P., \& Barrett, A. H. 1980, ApJ, 240, 84

Schuster, K. F., Boucher, C., Brunswig, W., et al. 2004, A\&A, 423, 1171

Sharp, D. H. 1984, Phys. D Nonlinear Phenomena, 12, 3

Shimajiri, Y., Kawabe, R., Takakuwa, S., et al. 2011, PASJ, 63, 105

Simón-Díaz, S., Herrero, A., Esteban, C., \& Najarro, F. 2006, A\&A, 448, 351

Sofia, U. J., Lauroesch, J. T., Meyer, D. M., \& Cartledge, S. I. B. 2004, ApJ, 605, 272

Spitzer, L., Jr. 1954, ApJ, 120, 1

Stahl, O., Kaufer, A., Rivinius, T., et al. 1996, A\&A, 312, 539

Teyssier, D., Hennebelle, P., \& Pérault, M. 2002, A\&A, 382, 624

Thackeray, A. D. 1950, MNRAS, 110, 524

Tremblin, P., Audit, E., Minier, V., \& Schneider, N. 2012, A\&A, 538, A31

Troland, T. H., Goss, W. M., Brogan, C. L., Crutcher, R. M., \& Roberts, D. A. 2016, ApJ, 825, 2

van der Werf, P. P., Goss, W. M., \& O’Dell, C. R. 2013, ApJ, 762, 101

van Dishoeck, E. F., \& Black, J. H. 1988, ApJ, 334, 771

Watson, W. D., Anicich, V. G., \& Huntress, W. T., J. 1976, ApJ, 205, L165

Weaver, R., McCray, R., Castor, J., Shapiro, P., \& Moore, R. 1977, ApJ, 218, 377

Wolfire, M. G., Hollenbach, D., \& McKee, C. F. 2010, ApJ, 716, 1191 


\section{Appendix A: Complementary tables and figures}

Table A.1. Offsets of the $\mathrm{CO}$ globules with respect to $\theta^{1}$ Ori $\mathrm{C}$ and line peak temperature (absolute calibration error of $\sim 15 \%$ ).

\begin{tabular}{rcccc}
\hline \hline & Offsets & \multicolumn{3}{c}{$T_{\mathrm{mb}, \mathrm{P}(\mathrm{K})}$} \\
Globule & & ${ }^{12} \mathrm{CO}(2-1)$ & ${ }^{13} \mathrm{CO}(2-1)$ & $\mathrm{C}^{18} \mathrm{O}(2-1)$ \\
\hline$\# 1$ & $\left(-825^{\prime \prime},-122^{\prime \prime}\right)$ & 3.1 & 0.3 & - \\
$\# 2$ & $\left(-878^{\prime \prime},-155^{\prime \prime}\right)$ & 5.3 & - & - \\
$\# 3$ & $\left(-999^{\prime \prime},-367^{\prime \prime}\right)$ & 8.8 & 0.8 & - \\
$\# 4$ & $\left(-1143^{\prime \prime},-1249^{\prime \prime}\right)$ & 14.6 & 0.8 & - \\
$\# 5$ & $\left(-1762^{\prime \prime},-654^{\prime \prime}\right)$ & 40.7 & 13.3 & 1.4 \\
$\# 6$ & $\left(-1108^{\prime \prime},-296^{\prime \prime}\right)$ & 3.4 & 0.3 & - \\
$\# 7$ & $\left(-992^{\prime \prime},-202^{\prime \prime}\right)$ & 40.8 & 8.9 & - \\
$\# 8$ & $\left(-1105^{\prime \prime},-54^{\prime \prime}\right)$ & 33.2 & 5.7 & - \\
$\# 9$ & $\left(-1023^{\prime \prime},-35^{\prime \prime}\right)$ & 26.3 & 2.2 & - \\
$\# 10$ & $\left(-841^{\prime \prime},+61^{\prime \prime}\right)$ & 52.5 & 15.5 & 1.4 \\
\hline
\end{tabular}

Notes. ${ }^{12} \mathrm{CO},{ }^{13} \mathrm{CO}$, and $\mathrm{C}^{18} \mathrm{O}(\mathrm{J}=2-1)$ lines convolved at a uniform angular and spectral resolution of $16^{\prime \prime}$ and $0.4 \mathrm{~km} \mathrm{~s}^{-1}$, respectively.

Table A.2. Line parameters of the CO globules obtained from Gaussian fits to the spectral component blueshifted from OMC-1 velocities.

\begin{tabular}{|c|c|c|c|c|c|c|c|c|c|}
\hline & $\begin{array}{l}\text { Vel. Centroid } \\
\quad\left(\mathrm{km} \mathrm{s}^{-1}\right)\end{array}$ & & & $\begin{array}{l}\text { Line width } \\
\left(\mathrm{km} \mathrm{s}^{-1}\right)\end{array}$ & & & $\begin{array}{l}\text { Line intensity } \\
\left(\mathrm{K} \mathrm{km} \mathrm{s}^{-1}\right)\end{array}$ & & \\
\hline Globule & [C II] & ${ }^{12} \mathrm{CO}$ & ${ }^{13} \mathrm{CO}$ & [C II $]$ & ${ }^{12} \mathrm{CO}$ & ${ }^{13} \mathrm{CO}$ & [C II $]$ & ${ }^{12} \mathrm{CO}$ & ${ }^{13} \mathrm{CO}$ \\
\hline$\# 1$ & $-2.1(0.3)$ & $-2.4(0.5)$ & $-2.3(0.5)$ & $5.4(0.6)$ & $1.4(0.1)$ & $1.3(0.5)$ & $32.4(2.8)$ & $4.8(3.2)$ & $0.5(0.2)$ \\
\hline$\# 2$ & $-1.2(0.2)$ & $-2.0(0.3)$ & - & $6.6(0.5)$ & $1.9(0.7)$ & - & $33.1(2.0)$ & $10.6(3.3)$ & - \\
\hline$\# 3$ & $-5.2(0.1)$ & $-5.4(0.1)$ & $-5.4(0.1)$ & $2.5(0.3)$ & $1.3(0.1)$ & $1.1(0.1)$ & $22.4(2.1)$ & $13.2(0.2)$ & $0.9(0.1)$ \\
\hline$\# 4$ & $+3.4(0.1)$ & $+3.7(0.1)$ & $+3.5(0.1)$ & $2.4(0.2)$ & $1.6(0.1)$ & $1.2(0.1)$ & $58.4(3.0)$ & $24.3(1.3)$ & $1.0(0.1)$ \\
\hline \#5 & $+6.0(0.1)$ & $+6.0(0.1)$ & $+6.4(0.1)$ & $6.0(0.2)$ & $3.1(0.1)$ & $2.0(0.1)$ & $112.0(2.9)$ & $135.3(0.2)$ & $28.7(0.1)$ \\
\hline$\# 6$ & $+1.6(0.1)$ & $+0.8(0.4)$ & $+1.9(0.1)$ & $2.8(0.2)$ & 1.1(0.3) & $1.2(0.6)$ & $47.7(2.3)$ & $3.9(1.3)$ & $0.4(0.1)$ \\
\hline$\# 7$ & $+5.4(0.1)$ & $+4.7(0.3)$ & $+4.7(0.1)$ & $3.5(0.2)$ & $1.5(0.3)$ & $1.1(0.1)$ & $80.8(5.7)$ & $64.1(2.6)$ & $10.2(0.1)$ \\
\hline \#8 & $+3.7(0.3)$ & $+3.8(0.3)$ & $+4.0(0.1)$ & $3.0(0.4)$ & $1.9(0.3)$ & $1.0(0.1)$ & $29.2(7.3)$ & $65.4(3.2)$ & $6.1(0.2)$ \\
\hline \#9 & $+2.7(0.2)$ & $+3.1(0.1)$ & $+3.1(0.1)$ & $2.5(0.3)$ & $1.5(0.1)$ & $1.4(0.1)$ & $18.7(4.3)$ & $41.5(0.2)$ & $3.2(0.2)$ \\
\hline$\# 10$ & $2.8(0.1)$ & $+2.7(0.1)$ & $+2.8(0.1)$ & $4.9(0.1)$ & $2.0(0.1)$ & $1.3(0.1)$ & $140.3(2.3)$ & $111.9(0.2)$ & $21.2(0.2)$ \\
\hline
\end{tabular}

Notes. [C II $158 \mu \mathrm{m},{ }^{12} \mathrm{CO}$, and ${ }^{13} \mathrm{CO}(J=2-1)$ lines convolved at a uniform angular and spectral resolution of $16^{\prime \prime}$ and $0.4 \mathrm{~km} \mathrm{~s}{ }^{-1}$, respectively.
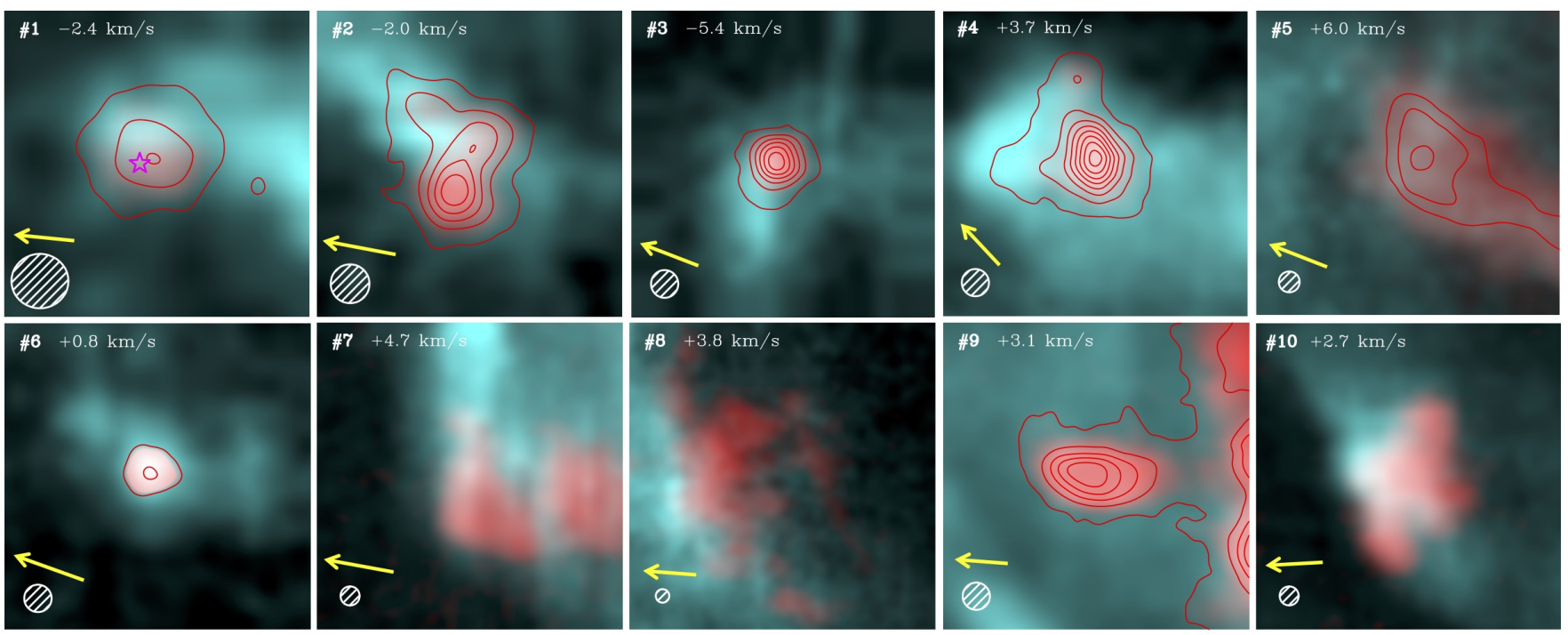

Fig. A.1. Gallery of detected CO globules. Same as Fig. 6 but showing the [C II] $158 \mu \mathrm{m}$ emission (bluish) integrated in exactly the same velocity range as the $\mathrm{CO}$ emission from each globule (reddish). The yellow arrows indicate the direction to star $\theta^{1}$ Ori $\mathrm{C}$ in the Trapezium. 


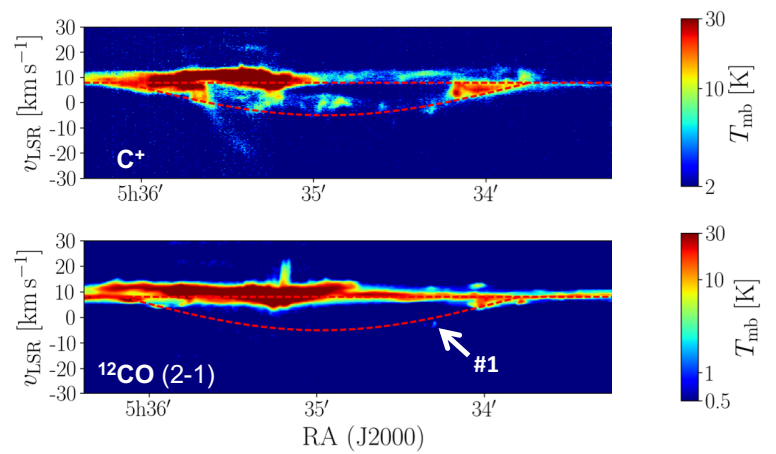

Fig. A.2. Same as Fig. 7 but for an east-west cut across the bubble at the declination of globule \#1 $\left(-5^{\circ} 25^{\prime} 24.23^{\prime \prime}\right)$.

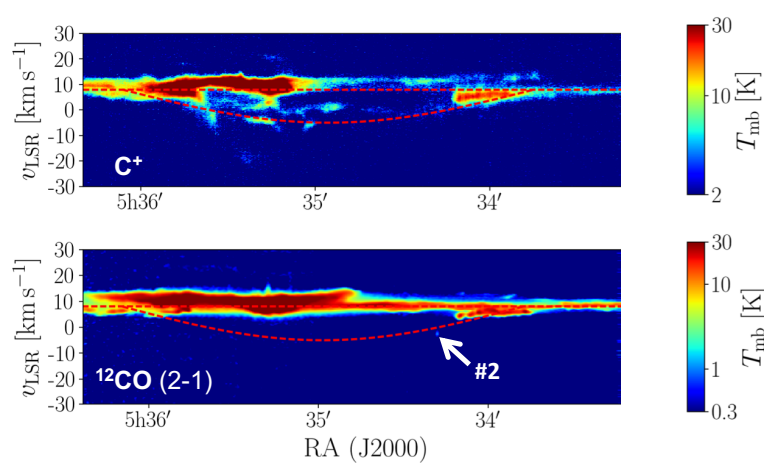

Fig. A.3. Same as Fig. 7 but for an east-west cut across the bubble at the declination of globule $\# 2\left(-5^{\circ} 25^{\prime} 57.52^{\prime \prime}\right)$.

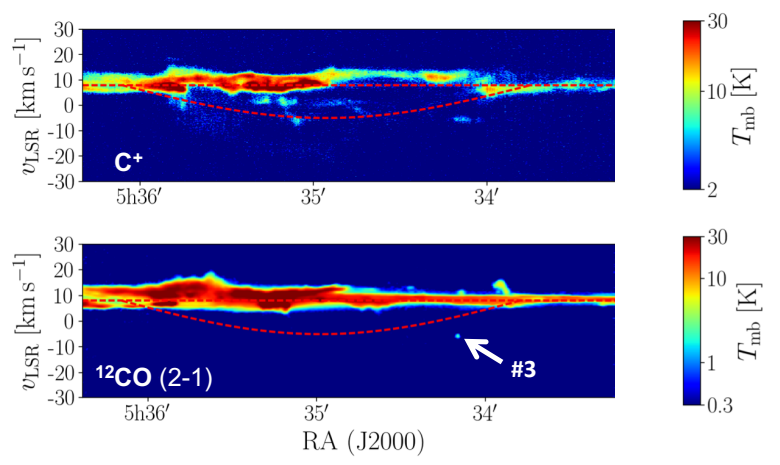

Fig. A.4. Same as Fig. 7 but for an east-west cut across the bubble at the declination of globule \#3 $\left(-5^{\circ} 29^{\prime} 29.40^{\prime \prime}\right)$.

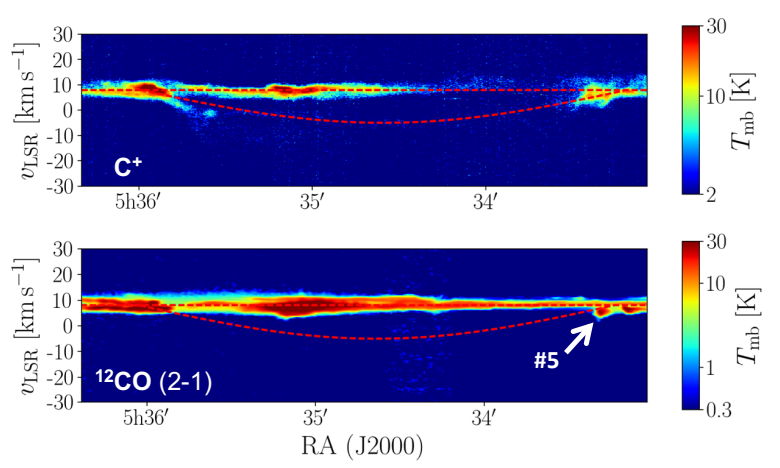

Fig. A.5. Same as Fig. 7 but for an east-west cut across the bubble at the declination of globule $\# 5\left(-5^{\circ} 34^{\prime} 16.29^{\prime \prime}\right)$.

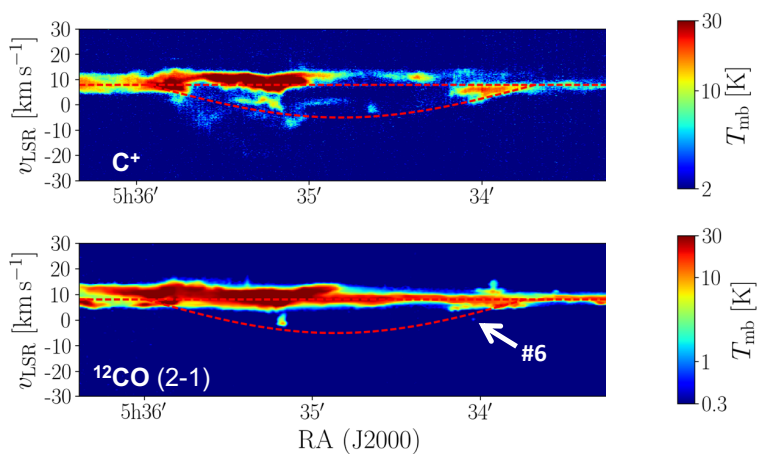

Fig. A.6. Same as Fig. 7 but for an east-west cut across the bubble at the declination of globule \#6 $\left(-5^{\circ} 28^{\prime} 18.58^{\prime \prime}\right)$.

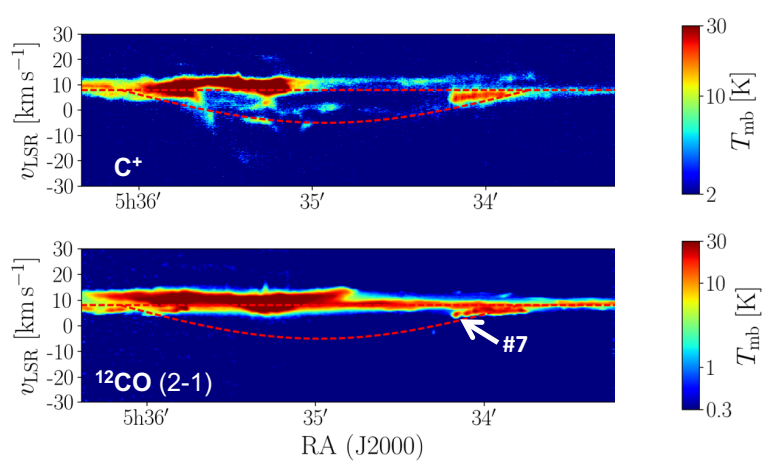

Fig. A.7. Same as Fig. 7 but for an east-west cut across the bubble at the declination of globule \#7 (-5 $\left.26^{\prime} 44.24^{\prime \prime}\right)$.

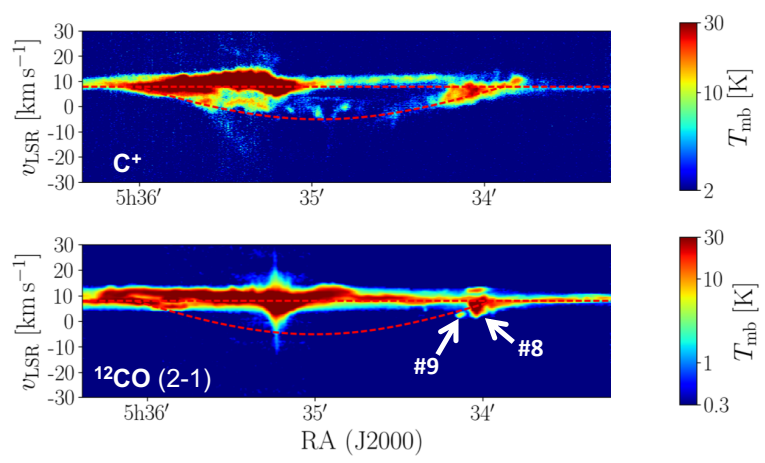

Fig. A.8. Same as Fig. 7 but for an east-west cut across the bubble at the declination of globules \#8 and \#9 $\left(-5^{\circ} 24^{\prime}\right)$.

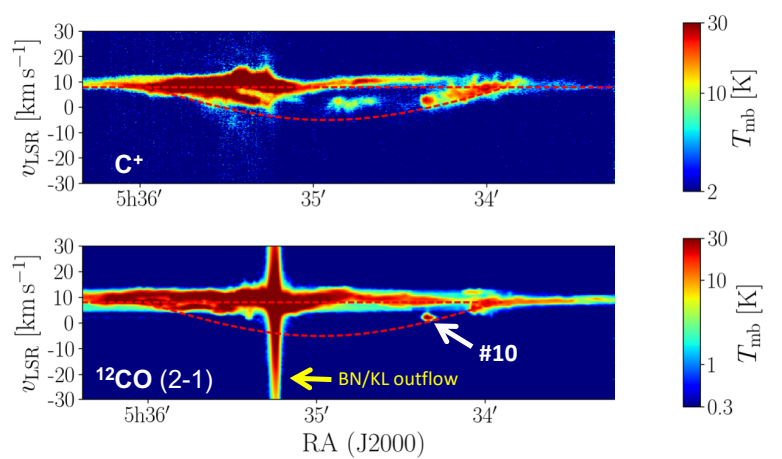

Fig. A.9. Same as Fig. 7 but for an east-west cut across the bubble at the declination of globule \#10 $\left(-5^{\circ} 22^{\prime} 21.93^{\prime \prime}\right)$. 

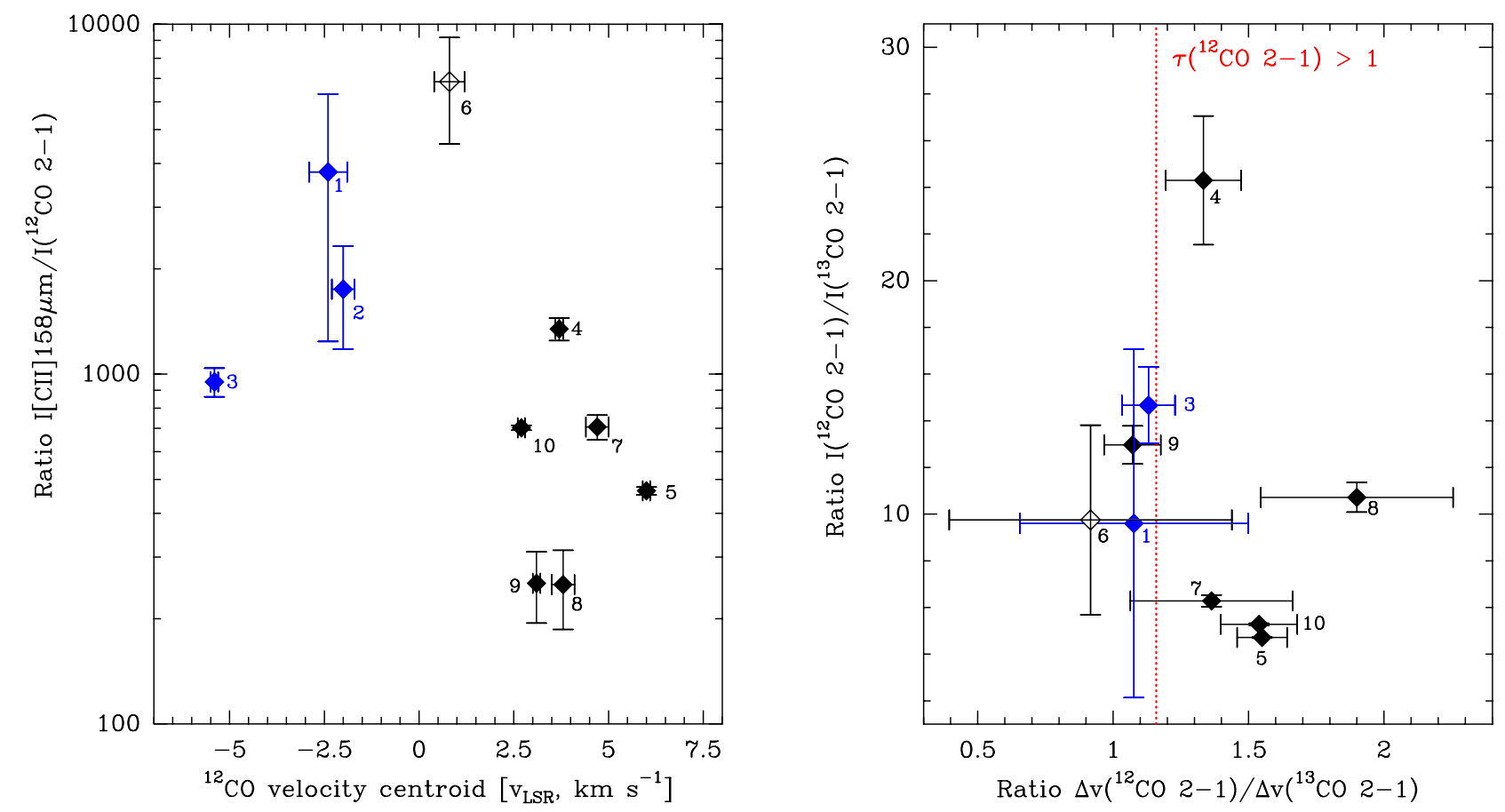

Fig. A.10. CO line emission from the globules. Blue (black) points correspond to the negative (positive) LSR velocity globules. Globule \#6 lies close in velocity to the negative- $v_{\mathrm{LSR}}$ globules and indeed shows similar emission properties. Left panel: $[\mathrm{C}$ II] $] 158 \mu \mathrm{m} /{ }^{12} \mathrm{CO}(2-1)$ line intensity ratio, in units of erg s${ }^{-1} \mathrm{~cm}^{-2} \mathrm{sr}^{-1}$, as a function of globule velocity centroid (as observed in ${ }^{12} \mathrm{CO}$ ). Right panel: ${ }^{12} \mathrm{CO} /{ }^{13} \mathrm{CO}(2-1)$ intensity ratio as a function of their line-width ratio. As gas column density raises, opacity broadening increases the width of ${ }^{12} \mathrm{CO}$ lines. The dashed red line approximately marks the $\tau\left({ }^{12} \mathrm{CO} 2-1\right)>1$ transition. Globules at the left of this line are not very thick in ${ }^{12} \mathrm{CO}(2-1)$, yet they show very low ${ }^{12} \mathrm{CO} /{ }^{13} \mathrm{CO}(2-1)$ line intensity ratios produced by ${ }^{13} \mathrm{CO}$ chemical fractionation.
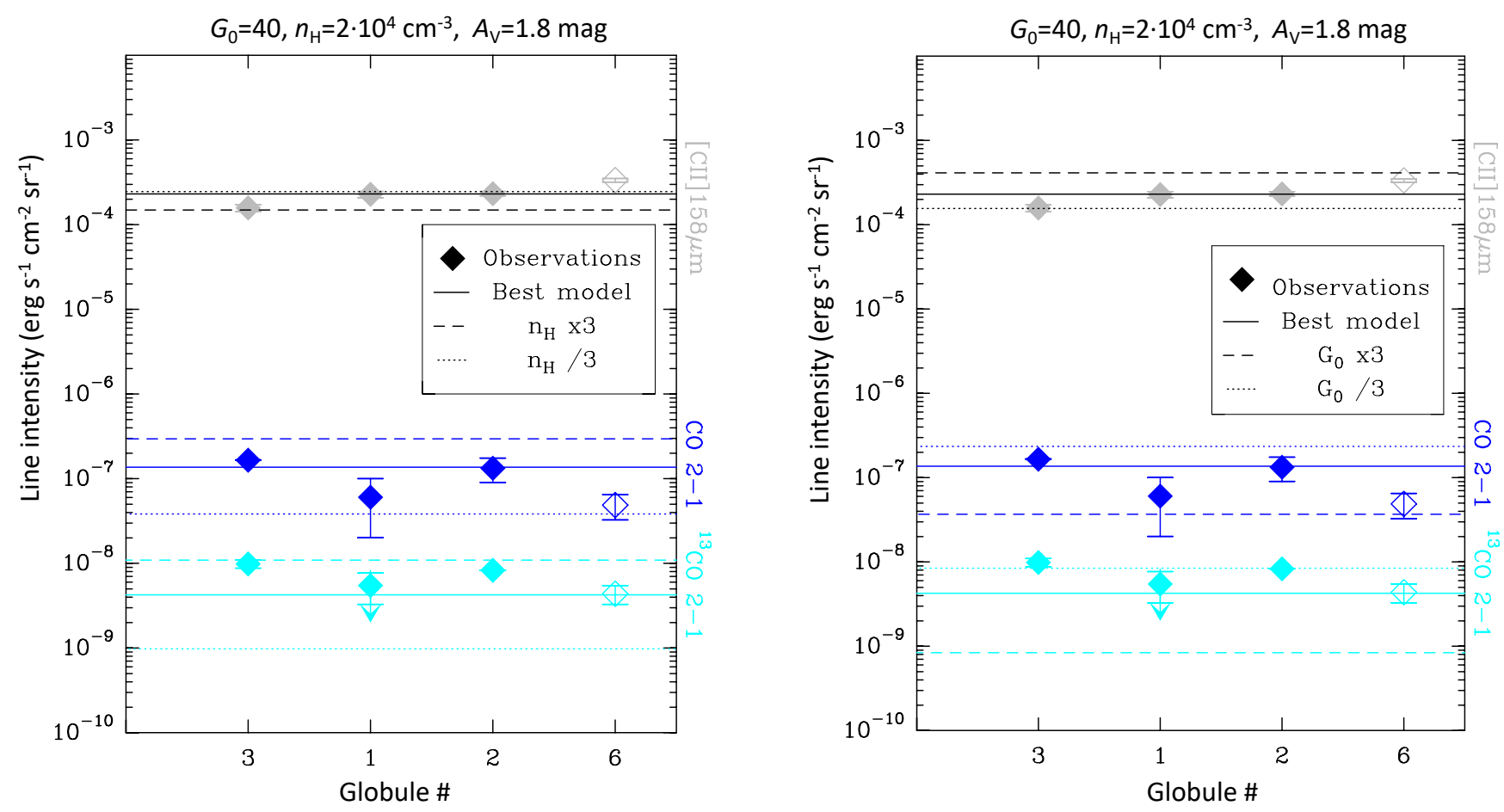

Fig. A.11. Comparison of PDR model predictions and observed line intensities from the negative- $v_{\mathrm{LSR}}$ globules. Error bars show the $1 \sigma$ uncertainty to the measured intensities obtained from a Gaussian fit to the observed lines. Globule \#6 lies close in LSR velocity and shows similar emission properties to the negative- $v_{\text {LSR }}$ globules. The velocity centroid of each globule increases from left to right in the $x$-axis (see Fig. 5). Left panel shows variations in the gas density whereas the right panel shows variations in $G_{0}$. 